\title{
A Review of Fiber-Reinforced Injection Molding: Flow Kinematics and Particle Orientation
}

\author{
M. Cengiz Altan \\ School of Aerospace and Mechanical Engineering \\ University of Oklahoma \\ Norman, OK 73019
}

(Received July 29, 1990)

\begin{abstract}
The existing flow and particle orientation models applicable to fiberreinforced injection molding are reviewed. After a brief description of injection molding, previous studies on the flow kinematics and fiber reinforcement are presented. Basics of Hele-Shaw flows are described including the commonly used viscosity models and fountain flow effects. Some of the existing models for particle orientation are analyzed with particular emphasis on the anisotropic description of the material system. Concentration regions for short fiber suspensions are defined and relevant constitutive equations are discussed. A few example solutions are also given which describe the three-dimensional orientation field for the filling of a sudden expansion cavity, depicting skin-core orientation structure.
\end{abstract}

\section{INTRODUCTION}

TN NUMEROUS ENGINEERING applications, the importance of the formation of ultra-high modulus fibers from polymeric liquid crystalline solutions, and the use of fibers as a reinforcing agent have long been recognized. With the current developments in the composite industry, the intensity of theoretical and experimental studies on fiber-reinforced molding operations has increased considerably. Currently, discontinuous glass or carbon fibers together with a polymeric resin (matrix material) are used most often to produce a composite material. This material system is usually formed by flow processes, such as injection, compression, or transfer molding. Depending on the flow characteristics, a flow induced orientation state is formed throughout the part, leading to an anisotropic part. For these short fiber-reinforced composites, several constitutive models are proposed to predict the elastic and thermal properties based on their microstructure. The general methodology calculates the elastic properties through averaging the unidirectional properties considering the fiber orientation distribution.

Halpin and coworkers suggest the laminate analogy $[1,2]$ where the composite is assumed to be made of several layers with different orientations. The relative 
thickness of each layer is taken to be proportional with the percentage of fibers at that orientation. On the other hand, a volume averaging is developed by several researchers [3-5] in which the unidirectional properties of several aggregates are averaged using an orientation distribution function. In all these models, the relationships suggested usually require information about the properties of the fiber, filler, and resin, the relative volume fraction of each constituent, the geometry of the fiber (i.e., fiber length, fiber diameter, effective aspect ratio $a_{p}$, etc.), and the description of the orientation field. The experiments performed [6,7] also show the dependence of elastic properties on the fiber orientation and the results are in good agreement with the proposed constitutive models. Most of the micromechanical constitutive models assume that the fiber orientation is known a priori. In order to make use of these models, the fiber orientation structure, which is induced during processing, must be determined thoroughly. Therefore, investigating the flow processes of the anisotropic fluids and understanding the driving mechanisms for the orientation structures are particularly important.

\section{INJECTION MOLDING}

\subsection{A Brief Description}

During the last three decades, polymer processing and related flow processes have been extensively investigated and are commonly used by the industry. Molding processes which are easily automated and suitable for high production volume, such as injection, compression, and transfer molding are largely utilized in daily life.

Several comprehensive studies have been published over the last 30 years. The most distinctive ones are, two texts on the fundamentals of polymer processing by Pearson and Middleman [8,9], an authoritative collection of works on the computational analysis of polymer processing [10], a text about the basics of injection molding [11], a descriptive collection about computational techniques on injection molding simulation [12], a recent book on polymer processing [13], and a text on reaction injection molding [14].

The injection molding operation is one of the most complex and, yet, commonly used polymer processing operations. In this process, the hot molten polymer is injected into a mold cavity with cold walls. As soon as the polymer is solidified in the cavity, it is removed and the cycle is repeated. The basic steps of injection molding operation are:

1. Melting the polymer with electric heaters and mixing it with a screw extruder in the reservoir

2. Injecting the molten polymer, usually with constant flow rate. The polymer melt is injected at relatively high speed from the nozzle to the sprue, which is the main flow channel to the mold cavity. Then, the polymer is distributed to the inlet gates through the sprue and runners. From these inlet gates the mold is filled.

3. Packing the filled cavity with high pressure to ensure that the mold is completely filled 
4. Heating or cooling for a short period of time in order to obtain minimum residual stresses and thermal shrinkage

5. Removing the part from the mold cavity.

\subsection{Studies on Flow Kinematics}

Mathematical and computational modeling of the injection molding process has numerous potential difficulties. The flow is transient with a moving boundary (free surface), the fluid is non-Newtonian and sometimes exhibits viscoelastic effects. Moreover, conduction and convection heat transfer take place in three dimensions, and a phase change occurs during the process. The flow is usually between thin gap cavities with complex planar shapes. The geometry of the mold can be either planar or three-dimensional; however, since the upper and lower boundaries of the domain are separated by a thin gap width, several simplifying assumptions can be employed related to the flow behavior.

Since the observations of Hele-Shaw [15] about the flow of water in thin planar cavities, a number of studies have been performed on the rigorous mathematical treatment of the problem and its applications to the injection molding processes. Hele-Shaw flows with free boundaries have been investigated by Saffman and Taylor $[16,17]$. Spencer and Gilmore [18-20] are among the first researchers to consider the dynamics of the injection molding problem. Richardson [21] studied the Hele-Shaw flows with a free boundary produced by the injection of a fluid. He considered a blob of Newtonian fluid in the narrow gap between two planar surfaces and estimated the motion of the initial fluid domain of any arbitrary shape, predicting its shape at subsequent times. Beyer and Spencer [22] have investigated the filling of an end-gated circular disk. Wu et al. [23] used power low fluid to solve the transient filling of a disk shaped cavity. Broyer et al. [24] adapted the flow analysis network (FAN) method, which was developed for die design, to the analysis of mold filling processes. They also considered the molecular orientation and packing effects on the molded parts. Kamal et al. [25] outlined the important stages in this process and analyzed their important characteristics. Later, Kuo and Kamal [26] analyzed the filling stage with a temperature and shear rate dependent viscosity model. Jetting phenomenon in injection mold filling is studied by Oda et al. [27]; they tried to characterize the two flow regimes, simple filling and jetting, and the transition between them.

A number of experimental techniques are employed by Bakerdjian and Kamal [28] to determine the three-dimensional variations of the properties such as density, heat shrinkage, birefringence and the tensile strength of injection molded thermoplastic rectangular parts. Although most of the numerical works described so far utilize finite difference methods, Gogos et al. [29] used the Marker-andCell (MAC) method to solve the problem including the fountain flow effects. MAC calculations are performed on Eulerian mesh with finite difference approximations. Their work showed good qualitative agreement with the experimental results involving "tracer resins" during the filling process. Hieber and Shen $[30,31]$ developed a hybrid finite element-finite difference scheme where they used finite elements for planar variables and employed finite difference discret- 
ization through the thickness. They presented the simulation of a two-gated plaque mold with unbalanced inlet conditions. The results were satisfactory in predicting weld-line formation and the pressure history at specific locations in the mold. Trafford et al. [32] and Subbiah et al. [33] applied a numerical grid generation technique to simulate the non-isothermal injection mold filling of complex planar shapes. The temperature calculations are performed using a transient three-dimensional energy equation. The experimental observations of fluid front locations and inlet pressure history agreed well with the predicted results [34]. Couniot and Crochet [35] presented a finite element scheme for the injection mold filling simulations. In this work, the domain is pre-meshed and, according to the fluid front movement, the local mesh is distorted to fit to the position of free surface.

In addition to the injection molding simulations, there have been a number of papers on the fluid front motion, particularly on the fountain flow that is observed in transient free surface flows. Behrens et al. [36] experimentally studied the motion of a fluid advancing in a tube. They also developed a numerical algorithm for the transient motion of the fluid. The numerical results were in good agreement with the experimental data over wide ranges of dimensionless groups such as Reynolds, Jeffery, and capillary numbers, Coyle et al. [37] and Blake [38] investigated the kinematics of fountain flow in mold filling. They constructed an apparatus which permits direct observation of the deformation of a fluid line experiencing fountain flow in a rectangular cavity. The experiments showed that shear-thinning behavior was relatively unimportant and the shape of the fluid front and flow kinematics did not change significantly. A finite element analysis was implemented for the solution of streamlines and deformation of tracer lines as a function of time, predicting V-shaped tracer formations behind the fluid front as depicted by numerous experiments. In a key note by Beris [39], the characteristic V-shapes were shown to develop behind the fluid front regardless of the fluid type, always pointing towards the inlet gate. Later, Mavridis et al. [40] worked on the effect of fountain flow on molecular orientation in injection molding. They presented a finite element solution for the fountain flow problem by using a multinode Leonov model.

\subsection{Studies on Fiber Reinforcement}

All of the above mentioned works investigated the kinematics and numerical treatment of the issues related to the injection molding. However, in addition to these works, characterization of the molding of reinforced plastics and analysis of the reinforcement structure were the main subject of several scientific studies. One of the earliest works on the orientation characterization of a molded part is presented by Woebcken [4I]. He studied the filler orientation in thermoset moldings and molecular orientation in thermoplastic injection molded parts to determine the effect of processing methods on structural characteristics. The use of a wide fan gate is suggested instead of a point gate in order to smooth out unwanted structural characteristics because of circular orientation (orientation perpendicular to the flow direction). The filling of a quarter disk, with a wall 
thickness of $4 \mathrm{~mm}$, is investigated with several short shots. The circular fiber orientation development (i.e., fibers aligning perpendicular to the flow direction) is observed when the surface is milled off about $0.5 \mathrm{~mm}$. In this work, shrinkage values of various compounds are investigated, and the values are found to be highly orientation dependent. Bell [42] worked on a method for characterizing the orientation of short fibers during flow. High concentration levels such as $45 \%$ to $63 \%$ volume fraction epoxy resin systems are considered. A glass tube rheometer was used with $1 / 2$ " diameter to observe the orientation formation during the flow. At these high concentrations, Woebcken observed plug flow along the rheometer and reported very high extrusion forces in comparison with the pressure needed for unfilled systems. Later, Tadmor [43] proposed a semi-quantitative model to analyze the molecular orientation in injection molding. He considered bead-and-spring macromolecular theory and incorporated a heat transfer model to account for the rapid skin solidification. He attributed the high surface alignment to the steady elongational flow in the advancing front and suggested that the core alignment is governed by the shear force behind the free surface.

In the case of glass fiber reinforcement, various aspects of orientation structure have been analyzed in a number of publications. Darlington et al. [44] performed several experiments on the end-gated fiber-reinforced molded disks. They concluded that, among several techniques, contact micro-radiography is the most versatile, being capable of giving consistent quantitative information about the fiber orientation distribution. Detailed analysis of the disks revealed that the fiber alignment changes significantly through the thickness. The tests carried out for uniaxial tensile creep confirmed that mechanical properties are highly dependent on fiber orientation.

Fiber orientation and rheological properties of Dough Molding Compounds (DMC) are analyzed by Gandhi and Burns [45] and by Owen and Whybrew [46]. The viscometric data by Gandhi and Burns showed a shear thinning behavior due to the presence of glass fibers. A squeeze flow between two parallel disks was used for the rheological characterization. They also found the aspect ratio to be an important factor for the viscosity where, at the same volume fraction, glass fibers with longer aspect ratio made the material system more viscous. Owen and Whybrew tested the tensile strength of transfer molded DMC compounds. The bars molded after a contraction exhibited more than twice the strength of bars molded after an expansion. For the material system, they also observed fiber buckling after expansion leading to undesirable properties. Chan et al. [47] reported an increase in jetting for the melts of polyethylene and polystyrene filled with glass fibers. It is shown that the addition of glass fibers decreases the extrudate swell and increases end pressure losses.

Bright et al. [48] analyzed the effect of injection speed on fiber orientation in short glass fiber filled polypropylene. The rheological properties of both glass fiber filled and unfilled polypropylene are studied in a capillary rheometer. They utilized contact micro-radiography to analyze the orientation patterns in simple strip molds; at high injection speed the fibers are observed to align transverse to the flow direction, whereas for low speeds the fibers are aligned parallel to the flow. Goettler [49] studied the mechanical property enhancement in short fiber 
composites during fabrication. He investigated the flow-induced orientation formation through runners, gates, and dies, and proposed techniques for optimizing directional stiffness and strength. The use of a fiber aspect ratio small enough to minimize bending and tangling is recommended. However, a large fiber aspect ratio may be required so that the hydrodynamic forces will result in fiber rotation. The effect of the thickness of the mold cavities is studied by Sanou et al. [50] in detail. The mold filling of a rectangular cavity of three different thicknesses is analyzed for unfilled and glass fiber filled polypropylene and polystyrene. The orientation of fibers in the cavity is examined by using a reflect type microscope for several short shots. The orientation is found to depend on cavity thickness, fiber concentration, and melt temperature. In the thinnest cavity, the fibers are observed to remain in the flow plane, and they are either along or perpendicular to the flow direction. The effect of the fountain flow on the advancing fluid is also observed where the fibers align along the parabolic profile across the thickness. The hydrodynamics and heat transfer that occur during molding of short fiber polymer composites are investigated by Kenig [51]. He qualitatively modelled the fiber orientation development during the mold filling and classified the mechanisms that contribute to the orientation formation. He concluded that there are basically four distinctive flow types influencing orientation structure:

1. Radial diverging flow near the inlet gates

2. Fountain flow at the advancing fluid front

3. Converging flow near the exit gates

4. Shear flow through the gap width

Vincent and Agassant [52] used optical microscopy to investigate the short glass fiber orientation structure in center gated molded discs. The different orientation formation between the core and the surface of the molding is quantified with an orientation distribution function. The injection molding of reinforced thermosets and fiber orientation formation are examined by Blanc et al. [53]. A relatively thicker core region and a thin skin layer without fibers are observed at low flow rates. The effect of cross-linking which leads to a pressure increase is predicted in this work. X-ray diffraction technique is used by Malik and Prud'homme [54] and Monge et al. [55] to determine the orientation of mica flakes in composites. Malik and Prud'homme found that the average orientation of mica flakes is strongly dependent on the size of the mica particles and the mode of preparation. Monge et al. observed the skin-core orientation of mica and talc platelets in injection molded polyamide 6.6.

\subsection{Flow Modeling: Hele-Shaw Flows}

\subsection{BASIC ASSUMPTIONS}

The modeling of the transient flow during the molding process requires fairly complex numerical techniques and immense computational power. Up to date, several numerical methods have been proposed to solve for the flow field inside a thin mold cavity. In all of these techniques, inertial terms are neglected and 
Hele-Shaw approximations are applied. The physical nature of the Hele-Shaw flow thus allows for the following simplifications:

1. Viscous forces are dominant, and inertial forces are neglected in the momentum equations.

2. Flow and dynamic forces in the gap-wise direction are neglected.

3. Shear stresses through the gap width are considered dominant (since the gap width is small), and the in-plane stresses are neglected.

4. The fluid is assumed to be incompressible.

\subsubsection{FLOW EQUATIONS}

Following the assumptions, the expressions that govern the fluid motion can be simplified to:

$$
\begin{aligned}
\text { continuity: } \quad \frac{\partial\left[h \bar{u}_{1}\left(x_{1}, x_{2}\right)\right]}{\partial x_{1}}+\frac{\partial\left[h \bar{u}_{2}\left(x_{1}, x_{2}\right)\right]}{\partial x_{2}} & =0 \\
x_{1} \text {-momentum: }-\frac{\partial p\left(x_{1}, x_{2}\right)}{\partial x_{1}}+\frac{\partial}{\partial x_{3}}\left[\eta_{v} \frac{\partial u_{1}\left(x_{1}, x_{2}, x_{3}\right)}{\partial x_{3}}\right] & =0 \\
x_{2} \text {-momentum: }-\frac{\partial p\left(x_{1}, x_{2}\right)}{\partial x_{2}}+\frac{\partial}{\partial x_{3}}\left[\eta_{v} \frac{\partial u_{2}\left(x_{1}, x_{2}, x_{3}\right)}{\partial x_{3}}\right] & =0
\end{aligned}
$$

where $u_{1}\left(x_{1}, x_{2}, x_{3}\right), u_{2}\left(x_{1}, x_{2}, x_{3}\right)$ are the velocity components of the three-dimensional flow in the $x_{1}$ and $x_{2}$ directions, $\bar{u}_{1}\left(x_{1}, x_{2}\right), \bar{u}_{2}\left(x_{1}, x_{2}\right)$ are the mean velocities averaged through the cavity gap width, $\eta_{v}$ is the fluid viscosity, and $h$ is the half gap width. After a little manipulation and using proper boundary conditions, a governing equation can be obtained with either pressure or the stream function as the dependent variable $[30,33]$.

$$
\begin{aligned}
& \frac{\partial}{\partial x_{1}}\left(S \frac{\partial p}{\partial x_{1}}\right)+\frac{\partial}{\partial x_{2}}\left(S \frac{\partial p}{\partial x_{2}}\right)=0 \\
& \frac{\partial}{\partial x_{1}}\left(\frac{1}{S} \frac{\partial \Psi}{\partial x_{1}}\right)+\frac{\partial}{\partial x_{2}}\left(\frac{1}{S} \frac{\partial \Psi}{\partial x_{2}}\right)=0
\end{aligned}
$$

where $S$ is a measure of fluidity and expressed as:

$$
S\left(x_{1}, x_{2}\right)=\int_{0}^{h} \frac{x_{3}^{2} d x_{3}}{\eta_{v}}
$$

The governing Equations (4), (5) are elliptic in nature, and boundary conditions need to be specified along all its edges. In the stream function formulation, the cavity walls can be represented by constant stream function values. For a 
thorough description of boundary conditions see Reference [34]. For isothermal, Newtonian flows the term $S\left(x_{1}, x_{2}\right)$ remains a constant, thus reducing the flow equation to Laplace's equation in terms of the stream function (or pressure). It is also important to note that during the filling process, the flow takes on various arbitrary shapes, and the solution of the governing equations over these irregular shapes demands the use of an appropriate numerical scheme.

Modeling of injection molding problems utilizing Hele-Shaw flow provides substantial simplifications to the problem. First, the flow is reduced to a twodimensional formulation using the average velocity components. Hence, a single governing equation in terms of stream function or pressure fully characterizes the flow. Second, three-dimensional shapes made of thin sections can be modeled with satisfactory accuracy. However, in this formulation no-slip boundary condition on the $x_{1}-x_{2}$ plane is violated leading to inaccurate predictions of flow kinematics near the boundaries. Moreover, in a geometrically complex shape, full three-dimensional velocity field may occur near $\mathrm{T}$ sections, inlet gates, and other intersecting planes. Adequate modeling of these regions and possible solution of complete three-dimensional velocity field where necessary, and then incorporating these results into an overall Hele-Shaw flow solution need to be further investigated.

\subsubsection{VISCOSITY MODELS}

In most molding operations, polymers exhibit highly non-Newtonian behavior. Their viscosity drastically changes as a function of temperature and shear rate. Consequently, during molding operations a shear-thinning viscosity is observed for most polymers such as polystyrene, polypropylene, etc. Several models have been developed to characterize the shear viscosity $\eta_{v}$ of the polymers. Some of the frequently used ones are described below:

\section{Power-Law Model}

$$
\begin{gathered}
\eta_{v}(\dot{\gamma}, T)=m(T) \dot{\gamma}^{n-1} \\
m(T)=m_{0} \exp \left[-\frac{A_{n}\left(T-T_{0}\right)}{T_{0}}\right]
\end{gathered}
$$

In this model, there are three parameters which need to be determined experimentally. These are:

1. $n$ : power-law index

2. $A_{n}$ : temperature sensitivity coefficient

3. $T_{0}$ : reference temperature

For more accurate representation, the coefficients $n$ and $m_{0}$ may also be functions of shear rate. However, power-law models yield unrealistic viscosities as shear rate approaches to zero or infinity. 


\section{Carreau Model}

$$
\eta_{v}=\eta_{\infty}+\left(\eta_{0}-\eta_{x}\right)\left[1+(\lambda \dot{\gamma})^{2}\right]^{(n-1) / 2}
$$

The Carreau model is a four-parameter equation which gives a shear-thinning viscosity at high shear rates with a Newtonian behavior at the zero shear rate limit. The model parameters are given as: zero shear rate viscosity $\eta_{0}$, infinite shear rate viscosity $\eta_{\infty}$, time constant $\lambda$ and power-law index $n$. These parameters can be used as functions of temperature to enhance the accuracy of the model.

\section{Cross-Arrhenius Model}

$$
\begin{gathered}
\eta_{v}=\frac{\eta_{0}(T)}{1+\left(\eta_{0} \dot{\gamma} / \tau^{*}\right)^{1-n}} \\
\eta_{0}(T)=B_{n} \exp \left(T_{b} / T\right)
\end{gathered}
$$

This is a five-parameter model, where $\eta_{0}$ is the zero shear rate viscosity, $T_{b}$ and $B_{b}$ are the temperature sensitivity coefficients for $\eta_{0}$, and $\tau^{*}$ is the shear stress level at which the $\eta_{v}$ exhibits a transition from Newtonian to power-law behavior.

\subsubsection{FOUNTAIN FLOW EFFECTS}

During mold filling, the fluid particles entering the cavity at the same time are translated by different velocities, depending on their location through the gap width. Since the fluid front moves with an average velocity $\overline{\mathbf{u}}$, some of the fluid particles will reach the fluid front and will be affected by the fountain flow. Therefore, when the cavity is filled, the particles will be distributed by the flow field into curves of constant residence times. In this section, within the framework of Blake [38], a simple model for space-time distribution of a cavity is presented.

For the present analysis, it is convenient to classify fluid particles into three categories:

1. Fluid particles that entered the domain first

2. Fluid particles that entered the mold later and did not reach the flow front

3. Fluid particles that entered the mold later and did reach the flow front

In order to continue the analysis, dimensionless cavity length $x_{1}^{\circ}$, dimensionless half gap width $x_{3}^{\circ}$, dimensionless velocity $u_{1}^{o}$, and dimensionless time $t^{\circ}$ are defined as:

$$
x_{1}^{o}=\frac{x_{1}}{L}
$$




$$
\begin{aligned}
& x_{3}^{o}=\frac{x_{3}}{h} \\
& u_{1}^{o}=\frac{u_{1}\left(x_{3}^{o}\right)}{\bar{u}_{1}} \\
& t^{o}=\frac{t}{t_{\mathrm{FILL}}}
\end{aligned}
$$

where $L$ is the cavity length. $h$ is the mold half gap width, $\bar{u}_{1}$ is the average velocity, and $t_{\mathrm{FILL}}$ is the total fill time.

Approximating the fountain flow as a single straight line moving with a velocity $\bar{u}_{1}$, there is a continuous fluid transfer from the faster moving regions to the slower moving regions. For a Newtonian fluid, the location $x_{3}^{o}$, at which this transition occurs (the point moving with $\bar{u}_{1}$ ) is found to be at:

$$
x_{3, r}^{o}=\frac{1}{\sqrt{3}}
$$

Then, the envelope of maximum residence time can be obtained as:

$$
x_{i_{m}}^{o}=\int_{0}^{1} u_{1}^{o}\left(x_{3_{m}}^{o}\right) d t^{o}=u_{1}^{o}\left(x_{3_{m}}^{o}\right) \quad x_{3_{\iota r}}^{o}<x_{3_{m !}}^{o}<1
$$

For a Newtonian fluid, the expression becomes:

$$
x_{i_{m}}^{o}=\frac{3}{2}\left(1-x_{3_{m}}^{o^{2}}\right) \quad x_{3_{1,}}^{o}<x_{3_{m}}^{o}<1
$$

Figure 1 shows this line of maximum residence time and the regions affected by the fountain flow. More complete treatment of the problem with solutions for non-Newtonian fluids can be obtained in Blake's work [38].

\section{MODELS FOR PARTICLE ORIENTATION}

In this section, the models for particle (fiber) motion in homogeneous flow fields (i.e., velocity fields with constant velocity gradients) and the commonly used constitutive models are reviewed. In a suspension system, the presence of particles will affect the rheological characteristics of the fluid. Depending on the orientation structure, the material system will exhibit different behavior for the prescribed boundary conditions. Therefore, a constitutive model is needed to describe how the stresses are generated due to the imposed deformations. Just as important are the motion of the fibers and the ways to describe the orientation structure. The equation of motion of fibers for a given flow field must be used to determine the fiber orientation state; then, this information has to be utilized in the proper constitutive model to describe material behavior. 


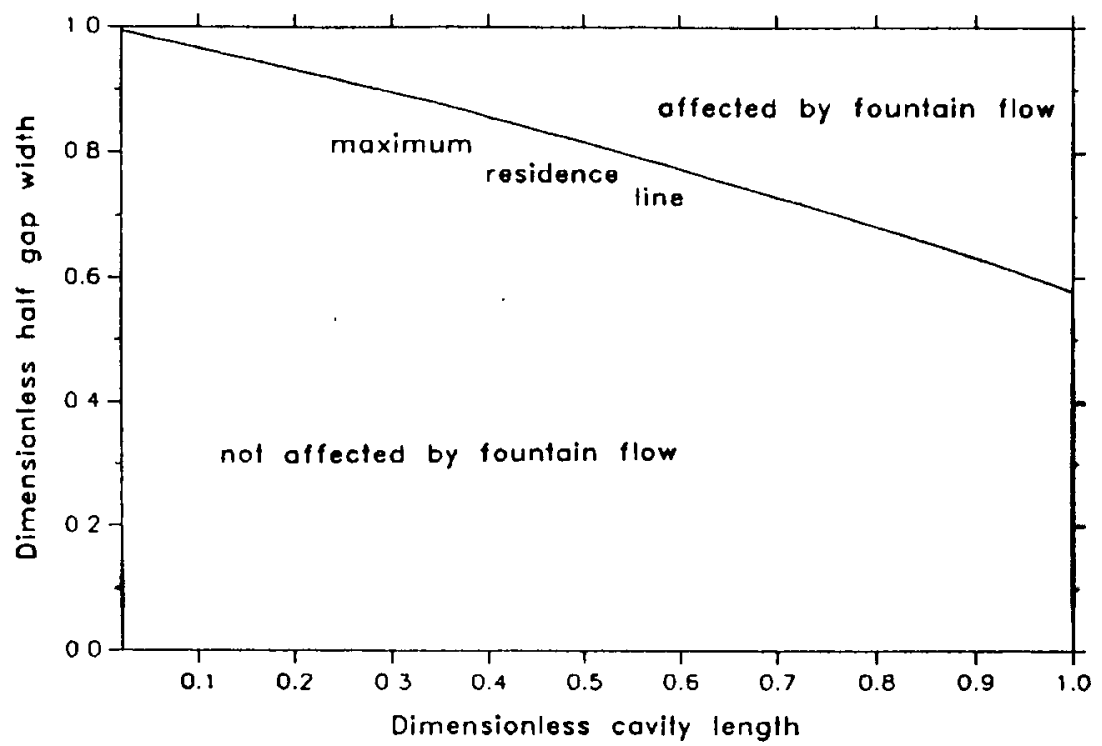

Figure 1. The regions affected from the fountain flow during the filling of a thin gap width cavity, after Blake's work [38].

Before analyzing particle motions and relevant constitutive models, it is essential to present some of the commonly used techniques to describe an orientation field. In the simplest case, a unit vector $\mathbf{p}$ is sufficient for orientation description. However, a vector (a scalar for 2-D) is not convenient for rheological characterization where the relation between deformation and generated stresses is the main interest. This brings up the issue of defining an orientation tensor. The task of defining tensorial quantities can be accomplished by the use of an orientation distribution function whose use is also supported by statistical models. The possible orientation descriptions and their properties are briefly listed below in the order of increasing complexity.

1. Orientation vector: $\mathbf{p}(\mathbf{x}, t)$

A unit vector $\mathbf{p}(\mathbf{x}, t)$ is used. For two-dimensional (i.e., planar orientations) cases, only a single angle $\phi$ is sufficient. For three-dimensional fields, spherical coordinates are utilized with two angles (i.e., $\theta, \phi$ ) specifying orientation field.

2. Orientation tensors: $S_{i j}, S_{i j k l}, \ldots$

Orientation tensors are concise, effective and convenient for constitutive models. Their definitions are based on the distribution function $\psi(\mathbf{p}, t)$ as

$$
S_{i j}=\int p_{i} p_{j} \psi(\mathbf{p}, t) d \mathbf{p}
$$




$$
S_{t j k l}=\int p_{\imath} p_{J} p_{k} p_{l} \psi(\mathbf{p}, t) d \mathbf{p}
$$

where $S_{\imath}$ and $S_{i j k l}$ are defined as second- and fourth-order orientation tensors. It should be noted that Equations (19) and (20) are valid for two- and threedimensional orientation fields and are completely symmetric (i.e., $S_{1112}=$ $S_{2111}$, etc.). An overall description of orientation tensors with possible applications can be found in Advani's work [56] in detail.

3. Distribution function $\psi(\mathbf{p}, t)$

Distribution function is described as the probability of having a particle between orientation $\mathbf{p}$ and $\mathbf{p}+d \mathbf{p}$. Although it is the most complete way of describing an orientation field, its calculation is mathematically complex and numerically intensive. Moreover, in most cases, its second- or fourth-order moments, if calculated properly, provide adequate information on orientation distribution.

\subsection{Equations for Particle Motion in Homogeneous Flow Fields}

\subsubsection{JEFFERY'S MODEL}

The motion of an ellipsoidal particle immersed in a viscous fluid is first studied by Jeffery [57]. He initially solved the equations of motion of the fluid surrounding the ellipsoidal particle. The inertia terms are neglected, and a Newtonian fluid is assumed in the analysis. No slip boundary condition is imposed at the particle surface, and an undisturbed homogeneous flow field is considered at large distances from the particle. The resulting Stoke's flow equations are solved with these boundary conditions. The velocity field and pressure distribution are obtained at the vicinity of the ellipsoid and used to calculate the forces acting on the particle. This formulation reveals two couples on the particle, one trying to impose the same rotation with the surrounding fluid, and the other trying to rotate the particle with its axes parallel to the principal axes of distortion of the surrounding flow. Having calculated the forces and the moments acting on the particle, Jeffery attempted to determine the equation of motion of the particle, which is not subject to any forces but only to the pressure of the surrounding fluid acting normal to the particle surface. He found a number of possible particle motions corresponding to different initial conditions. The rotations are periodic in nature, and the trajectories are now called "Jeffery orbits". It is also important to note that in Jeffery's analysis, the particle was large enough for Brownian motion to be negligible. At this point, it is appropriate to list the assumptions on which the Jeffery's equation is based:

- The particle is rigid, neutrally buoyant, axisymmetric, and large enough for Brownian motion to be negligible.

- The suspending fluid is Newtonian.

- Both particle and fluid inertia are negligible so that the fluid motion is determined by the Stoke's equation.

- The velocity gradients are constant over the particle length so that the particle is subjected to a homogeneous flow field. 


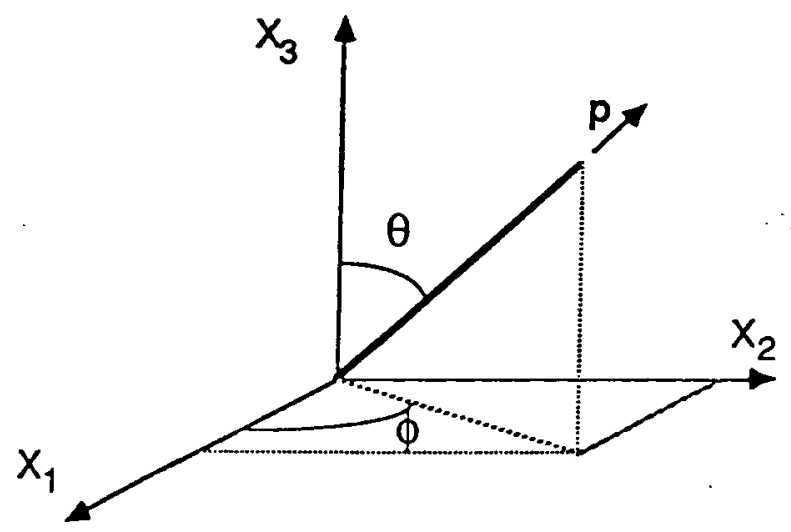

Figure 2. A descriptive figure for the three-dimensional fiber orientation in spherical coordinates.

In the analysis, the three principal semi-axes of the ellipsoid, i.e., $b_{1}, b_{2}, b_{3}$ were chosen to coincide with the axes of a Cartesian coordinate system $X_{1}^{o}, X_{2}^{o}$, $X_{3}^{o}$ at any time. Therefore, the ellipsoid surface can be represented as:

$$
\because \quad \frac{X_{1}^{0^{2}}}{b_{1}^{2}}+\frac{X_{2}^{0^{2}}}{b_{2}^{2}}+\frac{X_{3}^{0^{2}}}{b_{3}^{2}}=1
$$

Since the equation for a general ellipsoid is used, the solutions can easily be obtained for prolate or oblate ellipsoids as well as for a disk, a slender body, or a sphere at the limiting cases. The cases for different geometric shapes are given below:

$$
\begin{aligned}
\text { Elongated (prolate) ellipsoid: } & b_{1}>b_{2}=b_{3} \\
\text { Flattened (oblate) ellipsoid: } & b_{1}<b_{2}=b_{3} \\
\text { Disk: } & b_{1}=0 ; b_{2}=b_{3} \\
\text { Thin rod (slender body): } & b_{2}=b_{3} \rightarrow 0 \\
\text { Sphere: } & b_{1}=b_{2}=b_{3}
\end{aligned}
$$

Jeffery's equation was expressed in tensorial form by Giesekus [58] and Bretherton [59]. In addition, Brenner [60] derived similar equations for an arbitrary shaped particle. To describe the particle rotation, a spherical coordinate system with spherical angles $\phi$ and $\theta$ can conveniently be used as shown in Figure 2. If an ellipsoid of revolution with principal semi-axes $b_{1}$ and $b_{2}=b_{3}$ is taken, then the rate change of these spherical angles ( $\dot{\phi}$ and $\dot{\theta}$ ), which completely describes the three-dimensional particle rotation, can be expressed as [61]:

$$
\dot{\phi}=W_{23}-W_{31} \cos \phi \cot \theta-W_{12} \sin \phi \cot \theta
$$




$$
\begin{gathered}
+\lambda\left[-D_{12} \sin \phi \cot \theta+D_{23} \cos 2 \phi+D_{31} \cos \phi \cot \theta\right. \\
\left.-\frac{1}{2}\left(D_{22}-D_{33}\right) \sin 2 \phi\right] \\
\dot{\theta}=-W_{31} \sin \phi+W_{12} \cos \phi+\lambda\left[D_{12} \cos \phi \cos 2 \theta\right. \\
+\frac{1}{2} D_{23} \sin 2 \phi \sin 2 \theta+D_{31} \sin \phi \cos 2 \theta \\
\left.+\frac{1}{4}\left(D_{22}-D_{33}\right) \cos 2 \phi \sin 2 \theta+\frac{3}{4}\left(D_{22}+D_{33}\right) \sin 2 \theta\right] \\
\lambda=\frac{a_{p}-1}{a_{p}+1}
\end{gathered}
$$

where $a_{p}$ is the aspect ratio of the ellipsoid, and $W_{\imath}$ and $D_{\imath j}$ are the vorticity and strain rate tensors and defined as:

$$
\begin{aligned}
& W_{i j}=\frac{1}{2}\left(u_{j i}-u_{i j}\right) \\
& D_{i j}=\frac{1}{2}\left(u_{i j}+u_{j i}\right)
\end{aligned}
$$

Note that $u_{i j}=\left(\partial u_{i} / \partial x_{j}\right)$ is the velocity gradient tensor and summation over repeated indices is implied.

Analytic solutions of Equations (22-24) are not available except for a few simple flow kinematics. For example, in simple shear flow, the velocity field is given as:

$$
\mathbf{u}=\left(\begin{array}{l}
u_{1} \\
u_{2} \\
u_{3}
\end{array}\right)=\left(\begin{array}{c}
\dot{\gamma} x_{2} \\
0 \\
0
\end{array}\right)
$$

where $\dot{\gamma}$ is the shear rate. Hence, the strain rate tensor $\mathbf{D}$ and the vorticity tensor $\mathbf{W}$ become:

$$
\mathbf{D}=\left(\begin{array}{ccc}
0 & \dot{\gamma} / 2 & 0 \\
\dot{\gamma} / 2 & 0 & 0 \\
0 & 0 & 0
\end{array}\right)
$$




$$
\mathbf{W}=\left(\begin{array}{ccc}
0 & -\dot{\gamma} / 2 & 0 \\
\dot{\gamma} / 2 & 0 & 0 \\
0 & 0 & 0
\end{array}\right)
$$

For simple shear flow, the solution to Jeffery's equations is given as:

$$
\begin{gathered}
\dot{\phi}=\frac{\dot{\gamma}}{a_{p}^{2}+1}\left(a_{p}^{2} \cos ^{2} \phi+\sin ^{2} \phi\right) \\
\dot{\theta}=\frac{\dot{\gamma}\left(a_{p}^{2}-1\right)}{4\left(a_{p}^{2}+1\right)} \sin 2 \phi \sin 2 \theta
\end{gathered}
$$

Integration of Equation (29) shows that the rotation is periodic,

$$
\tan \phi=a_{p} \tan \left(\frac{\dot{\gamma} t}{a_{p}+1 / a_{p}}\right)
$$

and the period of rotation can be easily seen as:

$$
T=\frac{2 \pi}{\dot{\gamma}}\left(a_{p}+\frac{1}{a_{p}}\right)
$$

Equation (32) clearly shows that as $a_{p} \rightarrow \infty, T \rightarrow \infty$ indicating a stable steady state solution. Otherwise there is no steady state solution and, depending on its aspect ratio, the particle rotates with a non-uniform speed. As the aspect ratio becomes larger, the fiber remains oriented, most of the time, around the flow direction. Every half period, the fiber experiences a rapid change in the orientation by tumbling 180 degrees. Two-dimensional Jeffery's equations can be obtained for the case of planar orientations, that is, when the particle lies on the $x_{1}-x_{2}$ plane, and the velocity components $u_{1}$ and $u_{2}$ depend only upon the plane coordinates $x_{1}$ and $x_{2}$. Therefore, particle orientation is fully characterized by the orientation angle $\phi$ as shown in Figure 3.

For planar orientation, Jeffery's equation can be written as:

$$
\dot{\phi}=W_{12}+\lambda\left[D_{12} \cos 2 \phi-\frac{1}{2}\left(D_{11}-D_{22}\right) \sin 2 \phi\right]
$$

For a planar elongational flow, the flow field is given as:

$$
\mathbf{u}=\left(\begin{array}{l}
u_{1} \\
u_{2} \\
u_{3}
\end{array}\right)=\left(\begin{array}{c}
\dot{\epsilon} x_{1} \\
-\dot{\epsilon} x_{2} \\
0
\end{array}\right)
$$




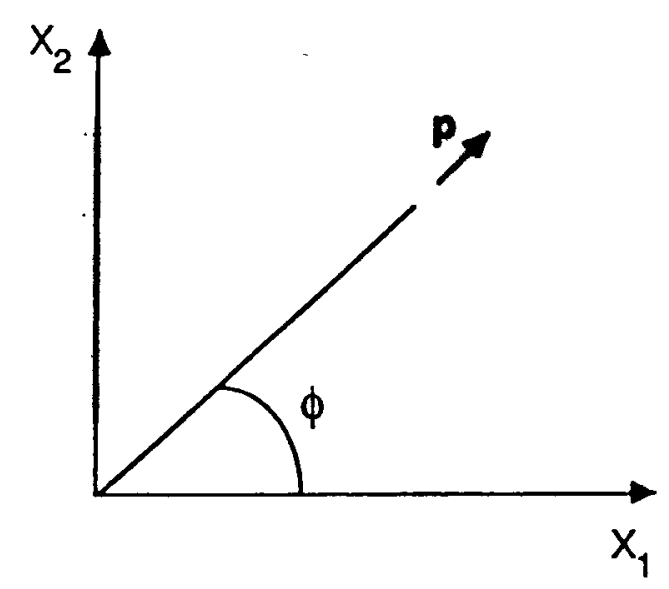

Figure 3. A descriptive figure for two-dimensional (planar) fiber orientation.

where $\dot{\epsilon}$ is the elongation rate. Consequently, the particle orientation is governed by:

$$
\dot{\phi}=-\lambda \dot{\epsilon} \sin 2 \phi
$$

The rotation of the particle $\dot{\phi}$ vanishes for $\phi=n \pi / 2, n=0,1,2, \ldots$, that is when the alignment is along the $x_{1}$ or $x_{2}$ axes. In fact, alignment along the $x_{2}$ direction represents an unstable equilibrium, and a small perturbation rotates the particle aligning it along the $x_{1}$ axis.

Jeffery's model can be extended by considering the presence of an external force field. Chaffey and Mason $[62,63]$ investigated the motion of ellipsoids in a combined shear and electric field. The experiments are performed in a Couette apparatus with an electric field, and the results compare favorably with the theoretical predictions. Furthermore, the model is applied to dilute suspensions by several other researchers. Okagawa et al. [64] analyzed the non-equilibrium state of the probability distribution of orientations of rigid fibers in simple shear flows. The intrinsic rheological properties, such as normal stress difference and transient viscosity, are obtained as averages with respect to the orientation distribution. Due to the unstable nature of the system, the properties are predicted to exhibit undamped oscillations. Experiments on sheared suspensions have shown that the orientation distribution and rheological properties do oscillate, but they are damped. These oscillations were observed to die away as a function of concentration level, indicating the importance of particle interactions even for dilute systems [65]. Goldsmith and Mason [66] studied the microrheology of dispersions extensively by using Jeffery's equations. The rotations of ellipsoids and stresses on particles in shear flow are investigated, and a comprehensive review on the experiments on reversibility and inertial effects are presented. Recently, Vincent and Aggasant [67] worked on the short fiber orientation in diverging 
flows. Jeffery's theoretical results are found to be in good agreement with the experimental observations of a single fiber flowing through a diverging die.

The numerical solution of Jeffery's equation for complex flows is performed by Givler et al. [68-70]. In these studies, a finite element numerical scheme for the determination of fiber orientation in dilute suspensions is developed and applied to several flow geometries. The solutions are obtained by solving the steady state velocity field in the given geometry, then by integrating the two-dimensional Jeffery's equations along the streamlines. Hence, the time derivative $\dot{\phi}$ reduces to:

$$
\dot{\phi}=V_{m} \frac{d \phi}{d s}
$$

where $V_{m}$ is the magnitude of the velocity vector, and $s$ is the arc length along the streamlines.

Other works on the fiber orientations in complex flows include papers by Hirch and by Squire et al. [71,72], which calculate fiber orientation by using Givler's approach and compare the results with the experimental data. Recently, Balasubramanyam [73] developed a numerical scheme for the determination of the shape of a fiber in flowing suspensions governed by Goddard-Huang equations [74].

It is important to keep in mind that Jeffery's equations describe the motion of a single ellipsoid particle in a viscous medium. For a suspension in which numerous fibers exist, the presence of fibers definitely affects the rheology of the system. Therefore, a constitutive model is needed to describe the complete behavior of the suspension. This constitutive model has to take into account the orientation structure in the fluid and must relate this information to the stresses generated in the suspension. Although Jeffery's equation is valid for zero volume fraction limit, the solutions provide some insight on how the fluid kinematics affect the fibers and even may give good qualitative results when applied to nondilute material systems.

\subsubsection{FOLGAR-TUCKER MODEL}

Folgar and Tucker [75,76] developed a mathematical model for concentrated suspensions. The model contains a phenomenological term to account for fiberfiber interactions. The analysis is based on Jeffery's equation, and an additional term is included so that the rotational diffusivity is governed by an interaction coefficient $C_{I}$ which is assumed to be the intrinsic property of the suspension. With the addition of the interaction coefficient, Jeffery's equation becomes:

$$
\dot{\phi}=W_{12}+\lambda\left[D_{12} \cos 2 \phi-\frac{1}{2}\left(D_{11}-D_{22}\right) \sin 2 \phi\right]-\frac{C_{r} \dot{\gamma}}{\psi_{\phi}} \frac{\partial \psi_{\phi}}{\partial \phi}
$$

where $\psi$ is the orientation distribution function and $\dot{\gamma}$ is the shear rate. The additional term $\left(C_{r} \dot{\gamma} / \psi_{\phi}\right)\left(\partial \psi_{\phi} / \partial \phi\right)$ is analogous to rotary Brownian motion of small particles. Therefore, the extra term implies a randomizing effect on the suspen- 
sion with a zero mean. The strength of this diffusivity depends on the magnitude of the interaction coefficient $C_{I}$ which needs to be determined experimentally. Folgar and Tucker proposed the use of an infinite aspect ratio $(\lambda=1)$ and very small interaction coefficients $\left(C_{I} \approx 10^{-1}-10^{-3}\right)$. Using Equation (37), the governing equation for distribution function, is obtained as:

$\frac{\partial \psi_{\phi}}{\partial t}+\frac{\partial}{\partial \phi}\left[\left(W_{12}+D_{12} \cos 2 \phi-\frac{1}{2}\left(D_{11}-D_{22} \sin 2 \phi\right) \psi_{\phi}-C_{r} \dot{\gamma} \frac{\partial \psi_{\phi}}{\partial \phi}\right]=0\right.$

As a result of the rotational diffusivity, the steady state orientation distribution is obtained regardless of the initial orientation distribution. Therefore, the reversibility of the Jeffery's model is not conserved with this additional term.

To verify the model, experiments were carried out in simple shear flow with nylon and polyester mono-filament fibers in silicon oil. The value of $C_{I}$ is found to be dependent on the fiber material, fiber volume fraction, and aspect ratio. However, a relation for a priori estimate of $C_{I}$ for a particular suspension system is not determined. Generally, good agreement is observed between the experiments and the theoretical predictions, provided that the interaction coefficient is determined carefully. The major reported disadvantage of this model is its prediction of rapid alignment at the inception of shear flow rather than the actual rate observed in the experiments.

Jackson et al. [77] applied the Folgar-Tucker model to spatially non-uniform flows and orientations. The orientation distribution of rigid short fibers are predicted in thin compression molded parts. Equation (38) is integrated along streamlines, and the two-dimensional distribution $\psi_{\phi}(\phi)$ is determined. Although the experimental data compared well with the numerical simulations, the rigorous determination of $C_{I}$ still remained unanswered. Nevertheless, the use of a rotational diffusion term in the governing equation for distribution function, even for the non-Brownian large fibers, provided insight into some of the observed complex phenomena such as:

- irreversible orientation formation resulting from particle-particle interactions

- disperse steady state orientation distribution, in contrast to the undamped oscillations or perfect steady state alignment

Folgar-Tucker model has also been implemented for the orientation predictions in short fiber-reinforced injection molded parts by Matsuoka et al. [78]. The governing equation is solved by using a finite difference method and velocity data obtained from the Hele-Shaw flow solution is used for planar orientation predictions. An orientation parameter is defined from the distribution function to describe the orientation field. The anisotropic thermal expansion coefficients are predicted from the calculated orientation field and compared with the experimental data. Numerous interaction coefficient values are tried for the best results and reasonable agreement is obtained with the measured quantities. 


\subsection{Concentration Regions}

In all rheological models proposed for fiber suspensions, the system behavior can be described within certain concentration regions.

\section{DILUTE REGION}

A dilute region is defined to be the one in which the spacing between a fiber and its neighbor is at leașt a fiber length. Thus, each fiber is free to rotate in any direction without interacting with another fiber. The volume necessary for each fiber to rotate freely is the volume of a sphere swept by a rotating fiber. Then, the maximum allowable volume fraction $\phi_{v}$ for a particle of length $l$ and diameter $d$ can be calculated as:

$$
\phi_{v} \leq\left(\frac{d}{l}\right)^{2}
$$

\section{SEMI-CONCENTRATED REGION}

In the semi-concentrated region, the distance between a fiber and its nearest neighbor, in a randomly oriented suspension, varies from a fiber length to a fiber diameter. Therefore, the volume fraction would be:

$$
\left(\frac{d}{l}\right)^{2} \leq \phi_{v} \leq\left(\frac{d}{l}\right)
$$

\section{CONCENTRATED REGION}

In the case of concentrated solutions, the average distance among fibers is less than a fiber diameter apart. For this region, the relative motion between the fibers and the fluid is very restricted. The volume fraction for this region becomes:

$$
\left(\frac{d}{l}\right) \leq \phi_{v}
$$

The concentration regions as a function of the volume fraction and the fiber aspect ratio are schematically shown in Figure 4. Note that the borders separating these regions are hypothetical and only serve for establishing guidelines for the different suspension behaviors.

\subsection{Molecular Models}

Molecular models are the constitutive descriptions at which the anisotropic fluid is viewed at a microscopic scale. At this microscopic scale, the particles are associated with the macromolecules of a polymer solution. The dynamics of polymeric materials have been studied for many years because of the widespread use of polymers in the plastics industry. A recent authoritative book on the theory of polymer dynamics by Doi and Edwards [79] covers this aspect thoroughly.

Among the several constitutive models, the most predominant one is developed 


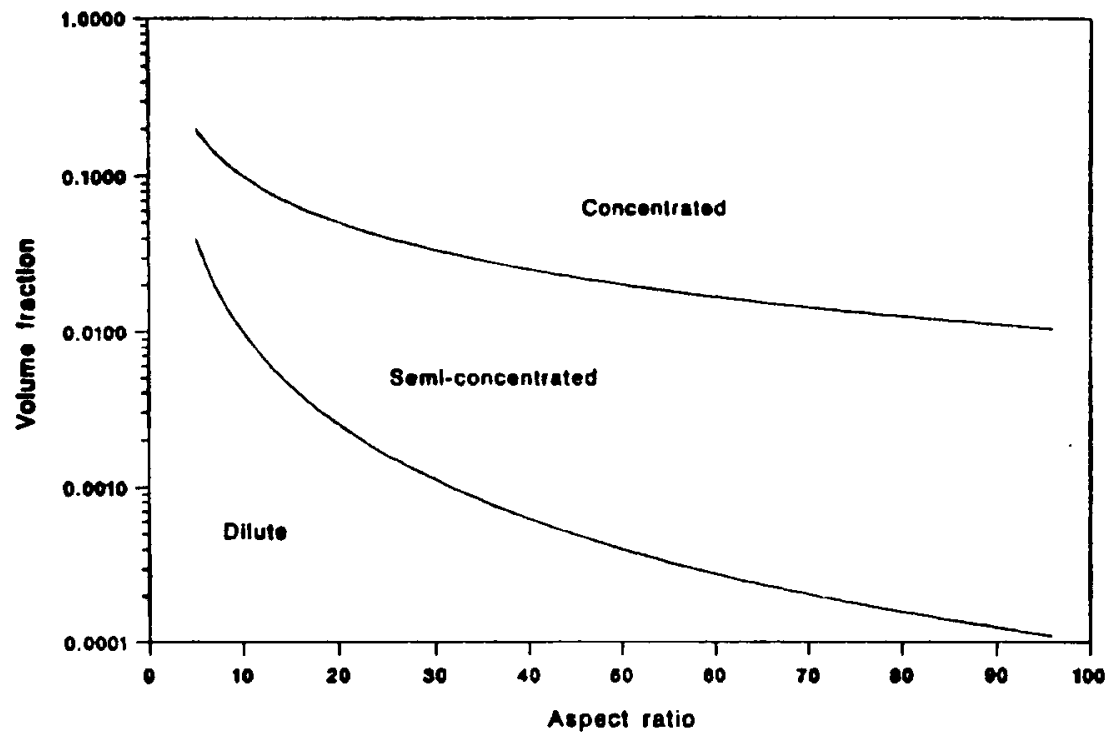

Figure 4. Concentration regions for fiber suspensions.

by Doi and Edwards [80-82] where the dynamics of rodlike macromolecules is investigated in concentrated solutions and applied to the rheology of rodlike polymers in isotropic and liquid crystalline phases. As defined in Section 3.2, the concentration regions can also be classified by using $n$ which is the number density of the particles (number of rods per unit volume).

$$
\text { Dilute region: } n<n_{1}=\frac{1}{l^{3}}
$$

Semi-concentrated region: $n_{1}=\frac{1}{l^{3}}<n<n_{2}=\frac{1}{d l^{2}}$

$$
\text { Concentrated region: } n>n_{2}=\frac{1}{d l^{2}}
$$

As the concentration is increased above a critical value $n^{*}$, the rods immediately align since there is not enough space for the rods to attain a random orientation. Doi and Edwards define the region between $n_{2}$ and $n^{*}$ as isotropic concentrated regime, and, if the concentration is greater than $n^{*}$, the suspension is called nematic liquid crystalline.

For semi-concentrated systems, the rod diameter is much smaller than the average distance between rods; therefore, longitudinal motion of particles is possible. However, transverse motions are blocked by the other particles and are quite 
small. Hence, the Doi-Edwards constitutive equation for semi-concentrated regions can be expressed as:

$$
\sigma_{i j}=n \xi_{f}<p_{i} p_{j} p_{k} p_{l}>u_{k l}+3 n k T_{a}\left(<p_{i} p_{j}>-\frac{1}{3} \delta_{i j}\right)
$$

where the first term is the viscous contribution of the stress tensor, and second term is the entropic (elastic) contribution, and $\langle\ldots\rangle$ represents the average over the distribution function. The constants $k$ and $T_{a}$ are the Boltzman constant and absolute temperature respectively, and $\xi_{f}$ is the friction coefficient as:

$$
\xi_{f}=\frac{\pi \mu_{f} l^{3}}{6 \ln (l / d)} ; \quad \text { with hydrodynamic interactions }
$$

or,

$$
\xi_{f}=\frac{\pi \mu_{f} l^{3}}{4} ; \quad \text { without hydrodynamic interactions }
$$

In the validity region of these expressions, the numerical values of Equation (43) and Equation (44) are not much different.

However, in the semi-concentrated regime, the viscous contribution to the stress tensor becomes smaller. Therefore, one can drop out the first term in Equation (42) for semi-concentrated regions.

$$
\left.\sigma_{t j}=3 n k T_{a}\left(<p_{t} p_{s}\right\rangle-\frac{1}{3} \delta_{t j}\right)
$$

Later, Doi extended the work for semi-concentrated regime to concentrated solutions of rod-like polymers in liquid crystalline phase by incorporating a Maier-Saupe potential [82]. In the extended form, the stress tensor for the concentrated regime can be expressed as:

$$
\sigma=\sigma^{\mathrm{e}}+\sigma^{2}+\sigma^{3}
$$

where $\sigma^{\mathrm{e}}$ is due to the elasticity, $\sigma^{\mathrm{v}}$ is due to the viscous effects, and $\sigma^{\mathrm{g}}$ is due to the solvent viscosity. These separate stress contributions can be expressed as:

$$
\begin{gathered}
\sigma_{i j}^{e}=3 n k T_{a}\left[S_{t j}-U S_{t k}\left(S_{j k}+\frac{1}{3} \delta_{j k}\right)-<p_{i} p_{j} p_{k} p_{l}>S_{k l}\right] \\
\sigma_{i j}^{v}=\eta \xi_{r} u_{k l}<p_{i} p_{j} p_{k} p_{l}> \\
\sigma_{i j}^{s}=\mu_{f}\left(u_{i j}+u_{j i}\right)
\end{gathered}
$$


and

$$
S_{i j}=\left\langle p_{i} p_{j}-\frac{1}{3} \delta_{i j}\right\rangle
$$

where $\xi_{r}$ is the rotational drag coefficient, $U$ is a dimensionless concentration parameter characterizing the intensity of the potential, and $\mu_{f}$ is the solvent viscosity.

Usually, at lower or intermediate shear rates, only $\sigma^{\mathrm{e}}$ is significant; therefore, most of the studies based on the Doi model concentrated on the first term representing the stress contribution associated with the free energy of the system. Doraiswamy and Metzner $[83,84]$ analyzed the stress contribution due to the solvent viscosity where at high shear rates this term is found to be significant. In addition, Doraiswamy proposed a two-phase heterogeneous model to account for the effects of the domain structures that often exist in polymeric liquid crystals.

Metzner and Prilutski [85] applied the Doi model to shear and extensional flows. The transient and steady state rheological properties are expressed in terms of order parameter $\mathbf{S}$. The theoretical results agreed well with the experimental observations for steady state and at low deformation rates; however, the model failed to depict the transient results. Marrucci extended Doi's theory to include the effect of a molecular field [86]. The final expression for the stress tensor is then linearized so that the expressions are obtained as equivalents to the Leslie coefficients. The Miesowicz viscosities are also estimated in the correct order. Recently, Edwards and Beris [87] studied the inherent assumptions of decoupling in the Doi model. The distribution function is calculated both from its exact definition and from its decoupled approximation. The exact results of the fourth order integral averages are compared with the approximated values, and considerable discrepancy is observed in some cases. The applicability of the Doi model to concentrated fiber suspensions was recently studied by Becraft [88]. The missing link is the fundamental derivation of a free energy potential for the concentrated fiber suspension so that the rotational diffusivity can be predicted based on the system parameters. The Doi model has been shown to fit the shear and extensional data over six and three decades of shear and extensional rates respectively, by using a two-parameter equation for each flow type. Its application to concentrated fiber suspensions seems promising.

\subsection{Continuum Models}

In this section, the continuum models for the anisotropic fluids are presented. In order to improve the molecular models explained in Section 3.3, the material has to be described specifically, including its defects and entanglement coupling. This will obviously reduce the applicability of the final model to the other anisotropic material systems. At this point, the need for more general models may arise without defining some material constants. Therefore, starting from the fundamental rules of constitutive theories, one can derive a general model with several phenomenological coefficients with little or no physical meaning attributed 
to them. The next step is to design and perform proper experiments which will help understanding the meaning of these constants. However, it is also possible to create useful constitutive equations from basic constitutive laws without using any structural or molecular information. The existing molecular theories can even be modified and enhanced by the simple use of a few constitutive rules. This section will concentrate mainly on the continuum theory of Ericksen and Leslie because of its generality and ability to predict several complex phenomena observed in anisotropic fluids.

The continuum theories on anisotropic fluids started with the works of Anzelius, Oseen, and Frank [89-91] on the theory of liquid crystals. These works analyzed mainly the equilibrium state of liquid crystals. First, the general structural theory of liquid crystals and the intermolecular forces were studied by Oseen. Then, Frank revised this work and dealt with the theory of curvatureelasticity in liquid crystals. In these early works, the molecular orientation is specified by a vector $\mathbf{n}$ and named as director. Starting from the sixties, Ericksen [92-97] and Leslie [98-101] developed a set of continuum formulations for the liquid crystalline fluids. In addition to the theories for nematic liquid crystals, other studies have also been performed for the continuum modeling of anisotropic fluids $[102,103]$.

\section{ERICKSEN-LESLIE MODEL}

The constitutive model developed by Ericksen and Leslie is one of the most general theories for anisotropic fluids. In this model, the effect of external forces such as electric or magnetic fields can also be accounted for. Initially, Ericksen proposed a set of equations which describes the additional stresses generated due to the orientation effects. These stresses, also known as Ericksen stresses, are isotropic functions of the velocity gradients, and the state of the director $\mathbf{n}$. The director $\mathbf{n}$ defines the preferred direction along the particle which is assumed to be a rigid rod at a spatial point $\mathbf{x}$.

The stresses $\sigma_{i}$, generated in the system can be expressed as:

$$
\begin{gathered}
\sigma_{\imath \jmath}=-p \delta_{\imath \jmath}+f_{\iota \jmath}\left(n_{k}, u_{m n}\right) \\
\dot{n}_{\imath}=g_{\imath}\left(n_{k}, u_{m n}\right)
\end{gathered}
$$

where $p$ is the isotropic pressure, $\delta_{i,}$ is the unit tensor, and $\dot{n}_{t}$ is the material derivative for a spatially non-uniform director field. It is obvious that the functions $f_{i j}$ and $g$, should preserve the same form under orthogonal transformation, and velocity gradients should be replaced by the strain rate tensor $D_{i j}$ in order to maintain symmetry. The material derivative $\dot{n}_{i}$ must also be changed by the Jaumann derivative defined as:

$$
\hat{n}_{\imath}=\dot{n}_{\imath}+W_{\imath \jmath} n_{j}=g_{\imath}\left(n_{k}, D_{m n}\right)
$$

The unknown function can be written in terms of two isotropic tensors, namely $D_{i}$, and the dyadic product of director $n_{i}$ as $N_{i j}=n_{i} n_{j}$. Based on these discus- 
sions, and avoiding nonlinearities by neglecting the higher order terms, Equations (51) and (53) can be written as:

$$
\sigma_{i j}=-p \delta_{i j}+2 \mu D_{i j}+\left(\mu_{1}+\mu_{2} D_{k m} n_{k} n_{m}\right) n_{i} n_{\jmath}+2 \mu_{3}\left(D_{\jmath k} n_{k} n_{2}+D_{i k} n_{k} n_{\jmath}\right)
$$

$$
\hat{n}_{\imath}=\dot{n}_{\imath}+W_{t,} n_{j}=\lambda\left(D_{i j} n_{j}-D_{k m} n_{k} n_{m} n_{\imath}\right)
$$

where $\mu$ 's and $\lambda$ are material constants.

Additionally, by using Equation (55), the solution for director $n_{\text {, }}$ is analyzed for simple shear flow, whose strain rate tensor $\mathbf{D}$ and vorticity tensor $\mathbf{W}$ are given before. Three coupled differential equations for the three components of the director is obtained from Equation (55).

$$
\begin{aligned}
& \dot{n}_{1}=\frac{1}{2} \dot{\gamma} n_{2}\left(\lambda+1-2 \lambda n_{1}^{2}\right) \\
& \dot{n}_{2}=\frac{1}{2} \dot{\gamma} n_{1}\left(\lambda+1-2 \lambda n_{2}^{2}\right) \\
& \dot{n}_{3}=-\lambda \dot{\gamma} n_{1} n_{2} n_{3}
\end{aligned}
$$

For this case, the steady state director orientation can easily be calculated by setting $\dot{n}_{t}=0$. It was shown that the steady state director orientation depends on $\lambda$ as:

$$
\frac{n_{2}}{n_{1}}=\tan \phi=\left[\frac{\lambda-1}{\lambda+1}\right]^{1 / 2}
$$

in which $\phi$ is the angle between the director and the free flow direction. Three different steady state orientations are predicted depending on the value of $\lambda$.

$$
\begin{array}{lll}
1<\lambda & 0<\phi<45 & n_{1} \neq n_{2}, n_{3}=0 \\
\lambda=1 & \phi=0 & n_{1}=1, n_{2}=n_{3}=0 \\
0<\lambda<1 & \text { Unstable } & n_{1} \neq n_{2} \neq n_{3}
\end{array}
$$

Depending on the value of $\lambda$, all the observed orientation behavior in simple shear flow can be obtained mathematically. However, the dependence of $\lambda$ to physical properties of the material is not defined. Obtaining realistic results for the director orientation, one can write the shear and normal stress components as:

$$
\sigma_{12}=\mu_{1} n_{1} n_{2}+\left[\mu+\mu_{2} n_{1}^{2} n_{2}^{2}+\mu_{3}\left(n_{1}^{2}+n_{2}^{2}\right)\right] \dot{\gamma}
$$




$$
\begin{gathered}
\sigma_{11}-\sigma_{22}=\mu_{1}\left(n_{1}^{2}-n_{2}^{2}\right)+\left(n_{1}^{2}-n_{2}^{2}\right) \mu_{2} n_{1} n_{2} \dot{\gamma} \\
\sigma_{22}-\sigma_{33}=\mu_{1}\left(n_{2}^{2}-n_{3}^{2}\right)+\left(n_{2}^{2}-n_{3}^{2}\right) \mu_{2} n_{1} n_{2} \dot{\gamma}+2 \mu_{3} n_{1} n_{2} \dot{\gamma}
\end{gathered}
$$

With the use of linear theory, it can easily be seen that the shear and normal stresses vary linearly with shear rate $\dot{\gamma}$. However, most of the liquid crystalline polymers exhibit shear rate dependent viscosity. Furthermore, in the case of $\mu_{1} \neq 0$, the model predicts a yield stress in both of the shear and normal stresses. In view of the fact that 5 independent constants need to be specified for a particular system ( $\lambda$ and $4 \mu$ 's), the application of this model to anisotropic materials is rather complex.

In the model described below, higher order terms can be considered for more realistic results. The non-linear form of these equations allows for the specification of shear rate dependent material properties. Thus, a shear rate dependent viscosity can be predicted by using the non-linear form. Similar to the Equations (59-61) for a simple shear flow, the non-linear form of this model would predict:

$$
\begin{aligned}
\sigma_{12} & =\alpha_{1} n_{1} n_{2}+\alpha_{2} \dot{\gamma} / 2+\alpha_{4} \dot{\gamma}\left(n_{1}^{2}+n_{2}^{2}\right) / 2 \\
& +1 / 2\left(\alpha_{5} \dot{\gamma}^{2}+n_{1} n_{2}\right) \\
\sigma_{11} & -\sigma_{22}=\alpha_{1}\left(n_{1}^{2}-n_{2}^{2}\right)+\alpha_{5} \dot{\gamma}^{2}\left(n_{1}^{2}-n_{2}^{2}\right) / 2 \\
\sigma_{22}-\sigma_{33} & =\alpha_{1}\left(n_{2}^{2}-n_{3}^{2}\right)+\alpha_{3} \dot{\gamma}^{2}+\alpha_{4} n_{1} n_{2} \dot{\gamma}+\alpha_{5} n_{2}^{2} \dot{\gamma}^{2} / 2
\end{aligned}
$$

For this case, there are six independent material constants that need to be specified ( $\lambda$ and $5 \alpha$ 's). For realistic results, one can assume $\mu_{1}=\alpha_{1}=0$, so that the model will not predict a yield stress in the absence of flow. For this case, using the Ericksen's linear model, steady state stress components for a simple shear flow can be expressed as:

$$
\begin{gathered}
\frac{\sigma_{12}}{\dot{\gamma}}=\mu+\frac{\mu_{3}}{2}+\frac{\lambda^{2}-1}{2 \lambda^{2}} \mu_{2} \\
\frac{\sigma_{11}-\sigma_{22}}{\dot{\gamma}}=\frac{\sqrt{\lambda^{2}-1}}{\lambda^{2}} \mu_{2} \\
\frac{\sigma_{22}-\sigma_{33}}{\dot{\gamma}}=\frac{\sqrt{\lambda^{2}-1}}{2 \lambda^{2}}\left[\lambda \mu_{3}+(\lambda-1) \mu_{2}\right]
\end{gathered}
$$

Uniaxial, planar, and biaxial extensional viscosities can also be expressed in terms of material constants. Using $n_{1}=1$ for the steady state:

$$
\begin{array}{ll}
\text { Uniaxial viscosity: } & \bar{\mu}_{u}=3 \mu+4 \mu_{3}+\mu_{2} \\
\text { Planar viscosities: } & \bar{\mu}_{p .1}=4 \mu+4 \mu_{3}+\mu_{2}
\end{array}
$$




$$
\begin{aligned}
\bar{\mu}_{p, 2} & =2 \mu \\
\text { Biaxial viscosity: } \quad \bar{\mu}_{b} & =6 \mu+4 \mu_{3}+\mu_{2}
\end{aligned}
$$

The model described above can be extended further to include magnetic and wall-induced distortional torque effects $[96,99,100]$. In the extended formulation, the stress tensor is no longer symmetric and is a linear function of $n_{i}, \dot{n}_{t}$, and $D_{i j}$. Viola and Baird [104] evaluated and compared the transient shear flow experiment of liquid crystalline polymers with the theoretical prediction of the Ericksen-Leslie model. They concluded that the stress growth response of LCPs is due to the change of the domain structure within the solvent. In this study, the stress overshoots observed at the inception of shear flow were attributed to the rearrangement of the initial domain structure. Another analytical study was presented by Duffy [105] on the orientation behavior of anisotropic fluids in extensional and squeezing flows. He considered a spatially uniform, but time dependent, orientation state and obtained some close form solutions for special cases in which the director motion is considered only in one plane.

Reviewing the voluminous literature on the continuum models, one can make use of these models for the general understanding of the behavior of anisotropic fluids. However, the main difficulty is to express the phenomenological constants in terms of relevant material properties. To accomplish this task, structural models can be used to provide a link between the continuum models and a particular material system.

\subsection{Structural Models}

The structural models provide a direct transition between the microstructural properties and the bulk (macroscopic) rheological behavior of a suspension. Therefore, it is possible to estimate the ambiguous phenomenological constants as a function of microstructural properties. Besides, these techniques provide an important means of calculating the rheological properties to be compared with experimental results. However, the analysis usually involves highly complex and laborious mathematics.

One of the earliest works on the expression of bulk stress in a suspension was presented by Batchelor [106], in which he developed a general constitutive equation for suspensions with arbitrarily shaped particles at arbitrary concentrations. However, direct application of this model is not possible due to the requirement of the motion and the local velocity field around each particle in the suspension. Later, Batchelor extended his early work and presented results for the stress generated in a non-dilute suspension of slender particles in elongational flows [107]. Since all the fibers are aligned along the flow direction in elongational flow, instead of considering all the particles separately, the analysis can be reduced to a single particle problem which is supposed to be immersed in an effective medium. This technique is sometimes referred to as a cell model or a self consistent continuum model. This effective medium is considered to be the continuum approximation of the hydrodynamic effects of the surrounding fibers on the particle 
considered. In Batchelor's early analysis, the bulk (average) stress due to the particles are given as [106]:

$$
\sigma_{i j}^{(p)}=\frac{1}{V_{o}} \Sigma \int_{A_{o}} \sigma_{\imath k} x, n_{k} d A
$$

where the integral is taken over the surface $A_{o}$ of a particle, and the summation is over the numerous particles in a volume $V_{o}$. Throughout the volume $V_{o}$, the fluid is considered to be statistically homogeneous. $\sigma_{t k} n_{k}$ is the force per unit area exerted on the particle surface by the surrounding fluid where the normal unit vector is denoted by $n_{k}$. The vector $x$, is measured from a fixed coordinate system to the area element $d A$ on the particle surface. It should be kept in mind that there should be some restrictions on volume $V_{o}$ over which the average is taken. $V_{o}$ should contain many particles, and the variation of local statistical properties of the suspension over $V_{o}$ should be negligible. Therefore, it is apparent that the linear dimensions of $V_{o}$ should be large compared to the average particle spacing.

Applying the "cell model" as proposed by Batchelor [107], Equation (72) can be reduced to a single particle equation.

$$
\sigma_{i j}^{(p)}=\frac{1}{V_{o}} \int_{A_{o}} \iint \Pi_{t k} n_{k} x_{j} \psi(\mathbf{r}, \mathbf{p}, t) d \mathbf{r} d \mathbf{p} d A
$$

where $\Pi_{t k}$ is the stress tensor describing the effective medium. A distribution function $\psi$ is introduced into Equation (73) since the analysis is performed for a single particle whose location and orientation is not known a priori at any time $t$. $\psi$ accounts for the probability of having the particle at point $\mathbf{r}$ with an orientation p at time $t$. Dinh and Armstrong [108] applied Batchelor's cell model idea and developed a constitutive model which is valid in the semi-concentrated region. Before presenting the details about the Dinh-Armstrong model, below is the list of assumptions used in the model as given in Dinh's work [109].

1. The suspending fluid is Newtonian.

2. The suspension is incompressible.

3. The fibers are rigid.

4. The fibers are large enough so that the Brownian motion is negligible.

5. The inertia effects are negligible.

6. There are no external force fields acting on the suspension.

7. The location of the centers of mass of the particles are homogeneously distributed throughout the suspension.

8. The hydrodynamic interactions between particles are modeled by considering a test fiber immersed in an effective medium. Therefore, this effective medium is considered to be the continuum approximation of the effect of other fibers.

9. The drag force on the particle is modeled by a line force along its major axis. 
Following these assumptions, the extra bulk stress tensor $\sigma_{i j}$ in a homogeneous flow field can be expressed as [109]:

$$
\sigma_{\imath,}^{(p)}=\mu_{f} \frac{\pi l^{3} n}{6 \ln \left(\frac{2 H}{d}\right)} \int u_{k l} p_{k} p_{l} p_{\imath} p, \psi(\mathbf{p}, t) d \mathbf{p}
$$

where

$n=$ number density of suspension

$l=$ fiber length

$d=$ fiber diameter

$u_{k l}=\left(\partial u_{k} / \partial x_{l}\right)$; components of the velocity gradient tensor

$p_{i}=i$-th component of the unit vector denoting the fiber orientation

$\psi(\mathbf{p}, t)=$ distribution function for the fiber orientation

$H=$ average distance from a given fiber to its nearest neighbor

$=(n l)^{-1 / 2}$ for aligned systems

$=\left(n l^{2}\right)^{-1}$ for random systems

$\mu_{f}=$ absolute viscosity of the fluid

The use of assumption 7 permits the calculation of the orientation distribution function $\psi$ with two independent variables which are $\mathbf{p}$ and $t$. The equation of motion for the fibers can be expressed in the form of Jeffery's equation with infinite fiber aspect ratio,

$$
\dot{p}_{i}=u_{i q} p_{q}-u_{k q} p_{q} p_{k} p_{i}
$$

Equation (75) is already built into Equation (74) and the coefficient of the integral in Equation (74) is determined by using the structural analysis of semiconcentrated suspensions [107].

The constitutive model can also be expressed in terms of the deformation gradient tensor as:

$$
\sigma_{i j}^{(p)}=\mu_{f} \frac{l^{3} n}{24 \ln \left(\frac{2 H}{d}\right)^{2}} u_{k l} \int \frac{p_{k} p_{i} p_{i} p_{j}}{\left(\Delta^{+} \Delta: \mathbf{p p}\right)^{\frac{3}{2}}} d \mathbf{p}
$$

where $\Delta$ is the deformation gradient tensor ( ${ }^{+}$implies its transpose) and defined as:

$$
\Delta_{i j}=\frac{\partial x_{i}^{\prime}}{\partial x_{j}}
$$


in which $x_{t}^{\prime}$ is the position vector at $t=0$ and $x_{\text {, }}$ is the position vector at time $t$. Average particle spacing and the dependence of $H$ on system parameters are recently investigated by Shaqfeh and Fredrickson [110].

Bibbo et al. [111] presented experimental data on the transient viscosity $\mu^{+}$at the inception of steady simple shear flow for semi-concentrated fiber suspensions. The resulting data are compared favorably with the theoretical predictions. In these experiments, a wide range of aspect ratios and volume fractions are covered within the semi-concentrated region. The experimental values of transient shear viscosity $\mu^{+}$are observed to be as much as 10 times of the shear viscosity of the suspending fluid. Since a Newtonian fluid is utilized, the rheological properties are found to be dependent only on total deformation, i.e., on total shear or total elongation. The effect of the walls on the measured rheological properties are also corrected in this study, improving the agreement with the model results. The major limitation of the initially proposed model may be the modeling of fibers with infinitesimal small diameters. Therefore, at steady state, i.e., when all the particles are aligned with the flow direction, the $\mu^{+}$drops down to $\mu_{f}$ so that the enhancement of the viscosity of the suspension due to the presence of particles is not observed. A correction function is proposed to account for the finite fiber thickness, and successful predictions are performed in comparison with the experimental data.

In a later study by Ganani and Powell [112], the macroscopic rheological properties of monodisperse fiber suspensions are analyzed. They considered glass fibers with a mean aspect ratio $\bar{a}_{p}$ of 24.3 with a mean length $\bar{l}$ of $267 \mu \mathrm{m}$. The volume fractions $\phi_{\nu}$ of $0.02,0.05$, and 0.08 were used in the experiments. The comparison of the experimental data with the Dinh-Armstrong model is performed, and they concluded that although the results confirm the basic approach of Dinh-Armstrong, there are some unknown mechanisms involved affecting the suspension behavior.

Recently, there have been additional studies on the behavior of fiber suspensions in complex flows. Shanker et al. [113] studied the rheology of fiber suspensions in nonhomogeneous flow fields. Papanastasiou and Alexandrou [114] worked on the isothermal extrusion of non-dilute fiber suspensions. They developed a numerical scheme in order to solve the velocity field during the extrusion process. They utilized Dinh-Armstrong constitutive equations and the resulting non-linear integro-differential equations were solved with a streamlined finite element technique. Their numerical predictions showed substantial difference between the dilute and non-dilute fiber suspensions in terms of processing conditions such as pressure drop, velocity field, and die swell.

Lipscomb et al. [115] studied the flow of fiber suspensions in sudden contractions. Ericksen's transversely isotropic continuum model [92] is applied to suspensions with large aspect ratio fibers as explained by Evans [116] (see also Hinch and Leal's work [117-118] for a detailed analysis). The material constants in Ericksen's model, Equations (54-55), are obtained by using the works of Giesekus and Hand for dilute systems based on volume averaging. This constitutive model is valid for dilute systems (non-interacting fibers) and in the limit of $a_{p} \rightarrow \infty$ the material properties are expressed as: 


$$
\begin{aligned}
& \mu_{0}=2 \mu_{f} \\
& \mu_{1}=\mu_{3}=0 \\
& \mu_{2}=\mu_{f} \frac{a_{p}^{2}}{\ln a_{p}} \\
& \lambda=1
\end{aligned}
$$

This form is very similar to the Dinh-Armstrong model which is valid for the semi-concentrated range. One can easily see that for Dinh-Armstrong the material coefficients can be written as:

$$
\begin{aligned}
& \mu_{0}=2 \mu_{f} \\
& \mu_{1}=\mu_{3}=0 \\
& \mu_{2}=\mu_{f} \frac{n l^{3}}{3 \ln \left(\frac{2 H}{d}\right)} \\
& \lambda=1
\end{aligned}
$$

The analysis shown above reveals that the same form of constitutive equations can be utilized for both dilute and semi-concentrated regions. The results obtained by using this model are compared with the experimental data taken from the flow of chopped-glass through axisymmetric sudden contraction. The increase in the size of the corner vertex is observed with the increase in concentration level, and numerical simulation is also shown to depict this observation very well. Lipscomb et al. [115] also concluded that there is not an internally consistent way of extending the existing models to incorporate long-range interactions that occur in concentrated suspensions. Following Lipscomb`s work, Chiba et al. [119] also presented numerical solutions for the flow of dilute fiber suspensions through an axisymmetric contraction. Later, Rosenberg et al. [120] implemented a finite element technique in which the constitutive equation is solved by an integration scheme along the flow streamlines. Since their technique is convenient for non-recirculating flows, simulations of planar extrusion and falling-ball rheometry are performed. Altan et al. [121] studied the flow of fiber suspensions in planar channels by using Dinh-Armstrong model. The change in the flow kinematics by the sudden introduction of random particles into the flow is investigated. In all the above-mentioned studies utilizing constitutive models, considerable changes are observed in the flow behavior due to presence of particles. 


\section{NUMERICAL ORIENTATION PREDICTIONS}

At the present time, the implementation of an anisotropic constitutive model on the modeling fiber-reinforced injection molding has not been studied and considerable potential difficulties are anticipated in such an attempt. The state-of-the-art computations related to injection molding utilize Jeffery's equation or its versions, and neither solidification nor fountain flow effects on the orientation field are taken into account. Therefore, the available solutions can be best described by the orientation field calculations for a quasi-steady state Hele-Shaw flow at zero volume fraction limit. Despite these limiting factors, predictions based on Jeffery's equation are capable of producing qualitatively satisfactory results $[71,122]$.

Along these lines, the applicability of Jeffery's equation to injection molding problems, and the validity of decoupling the flow kinematics and orientation field are studied by Tucker [123]. He has attempted to characterize the flow regimes for fiber suspensions in narrow gaps. The order-of-magnitude analysis related to velocity, stress, and fiber orientation revealed four distinctive regimes in which flow kinematics and orientation field behavior vary ranging from plug flow to parabolic fully developed profile. These regimes are shown to be dependent on the gap geometry, fiber orientation, and concentration.

Recently, Altan et al. [124] developed a numerical technique to determine the three-dimensional orientation structure in complex flows. This method is applied to Hele-Shaw flows by introducing a number of fiber tracer points from the inlet section at a specified rate. Each fiber location is traced during the flow and along these determined particle paths, independent components of the fourth-order orientation tensor are solved, describing orientation state. Although the evolution equation of orientation structure (i.e., fourth-order orientation tensor) is obtained from Jeffery's equation with infinite aspect ratio, the model in its given form is used in several constitutive models described in Section 3. Altan et al. [124] also proposed a convenient way of presenting orientation calculations by using threedimensional orientation ellipsoids. The proposed technique makes it possible to analyze planar, longitudinal, and transverse orientation structure simultaneously with relative ease. A more complete analysis and the ways to describe orientation field are presented in Altan's work [125,126]. Later, Friedrichs et al. [127] also presented three-dimensional orientation predictions at three different layers of an ASTM D638 tensile test bar. The orientation figures clearly showed the complex three-dimensional orientation field with skin-core structure.

In this section, an example of a 1-4 sudden expansion cavity is presented as described in Reference [126]. The domain has an overall length of $8.5 \mathrm{~cm}$, and the simulation is stopped when the flow front is at $6.88 \mathrm{~cm}$. The inlet width is taken to be $0.6 \mathrm{~cm}$ long and $1 \mathrm{~cm}$ wide before the expansion. A half cavity thickness of $h=0.2 \mathrm{~cm}$ and an average inlet speed of $30 \mathrm{~cm} / \mathrm{s}$ is used. At $t=0.91 \mathrm{~s}$ (when the flow is at $6.88 \mathrm{~cm}$ downstream), 7 sets of fibers are already introduced into the cavity. In Figures 5-7, the planar fiber orientations are shown at three levels along the gap width, i.e., $x_{3}=0$ (midplane), $x_{3}=0.3 h$, and $x_{3}=0.5 h$. In these figures, the planar projections of the predicted three-dimensional ellip- 
soids are shown. Circles represent a random orientation whereas lines indicate a complete alignment.

In Figure 5, the midplane orientation results are shown to have radial distribution throughout the cavity except near the walls where the fibers align along the domain boundaries. This result is in agreement with the experimental observations discussed in Section 2.3. The transition from the core orientation to skin orientation is observed in Figure 6 . Near the inlet gate, the extensional flow due to sudden expansion is found to be dominant; however, towards the end of the mold, the fibers are observed to be aligned along the flow direction due to the shearing effects through the gap width. At the upper layer towards the cavity wall, very high alignment is observed along the flow direction as shown in Figure 7.

\section{CONCLUDING REMARKS AND RECOMMENDATIONS FOR FUTURE WORK}

The investigation of the behavior of short fibers in flow processes such as injection molding is very challenging but yet an interesting topic. The problem has several potential difficulties at every stage and needs more scientific research in order to enhance our present understanding about these material systems. In this paper, a general review on the constitutive models describing the rheology of fiber suspensions is presented with an emphasis on the fiber orientation characterization and prediction in injection molding. The analysis of different models reveals that the commonly used techniques contain numerous limiting assumptions. More accurate models, supported by fundamental constitutive theories and experimental verification, are needed to describe the fiber motion in the concentrated systems. Currently, the proposed constitutive models are evaluated only for very simple flow types and their use for complex flows remains to be a major challenge. Future works on developing models which are convenient and useful for complex flows are needed.

At the present time, three-dimensional transient fiber orientation predictions for Hele-Shaw flows for the zero volume fraction limit is the most complete treatment of the fiber-reinforced injection molding problems. The three-dimensional orientation ellipsoids are proved to be useful for the graphical representation of the fiber orientation in the complex geometries. The effect of the specified initial condition for fiber orientation is not found to be significant since the fluid kinematics quickly takes over the effect of initial condition. The three-dimensional results for a sudden expansion clearly show the skin-core structure of the fiber orientation. At the midplane, the fibers are found to be perpendicular to the flow direction, whereas, at the layers closer to upper and bottom cavity walls, almost perfect alignment with the flow is observed. Near the side walls, very high alignment is predicted which is consistent with the experimental observations. However, the quantitative match may not be excellent for injection moldings since some of the important issues which may affect fiber orientation considerably are neglected. Among these, the most important ones may be the fountain flow effect and the rapid solidification of the material. Future work is definitely needed for the analysis of the fountain flow and its effects on the fiber orientation. The effect 


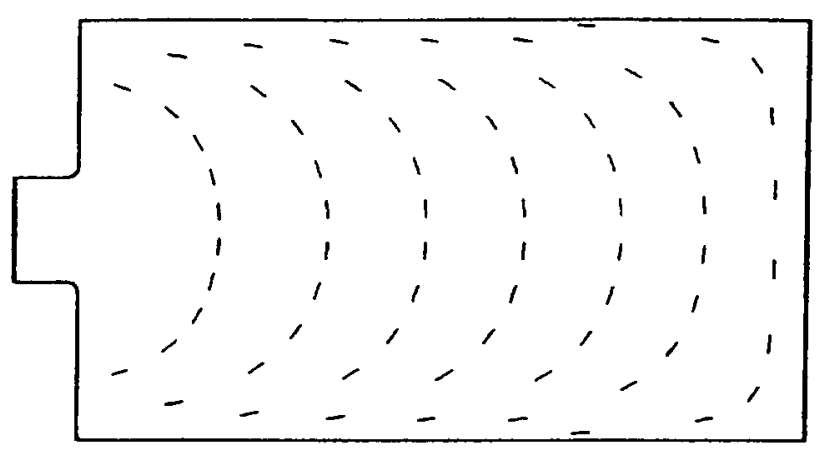

Figure 5. The planar fiber orientation at $x_{3}=0.0$ (midplane).

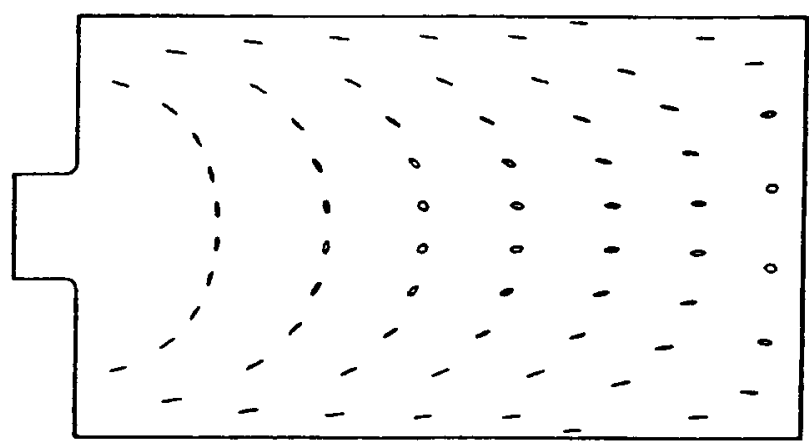

Figure 6. The planar fiber orientation at $x_{3}=0.3 \mathrm{~h}$.

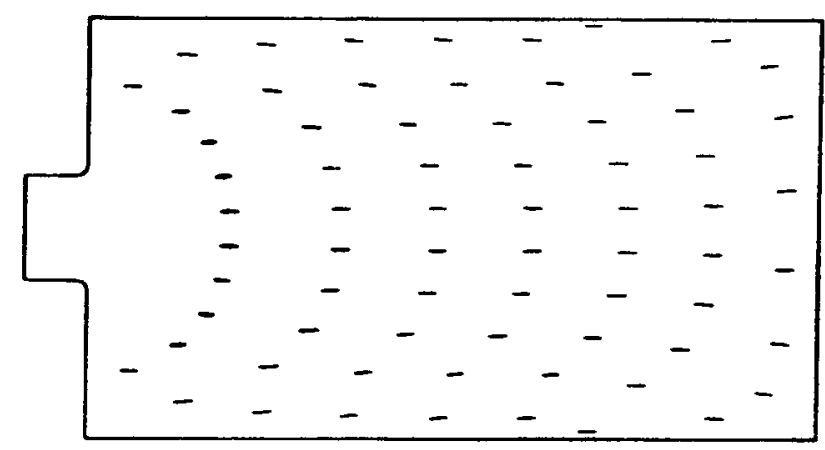

Figure 7. The planar fiber orientation at $x_{3}=0.5 \mathrm{~h}$. 
of the packing stage is also not known, but a number of claims related to fiber orientation do exist which require more investigation. In addition, the effects of fiber aspect ratio distribution, fiber migration, and buckling together with the fiber degradation are issues which have not been studied thoroughly. There is also little work on the modeling of fiber orientation in non-homogeneous flows where the fiber experiences significant velocity gradient variation over its length. The relation between this non-homogeneous effect and fiber bending, migration, and breakage is still open to further investigation.

\section{REFERENCES}

1. Halpin, J. C., K. Jerine and J. M. Whitney. 1971. "The Laminate Analogy for Two- and ThreeDimensional Composite Materials", J. Composite Materials, 5:36.

2. Halpin, J. C. and N. J. Pagano. 1969. "The Laminate Approximation for Randomly Oriented Fibrous Composites", J. Composite Materials, 3:732.

3. Christensen, R. M. and F. M. Waals. 1972. "Effective Stiffness of Randomly Oriented Fiber Composites", J. Composite Materials, 6:518.

4. Ghesquiere, A. and J. C. Bauwens. 1976. "Theoretical Models for the Elastic Behavior of Composites Reinforced with Short Fibers", J. Appl. Polymer Sci.. 20:891.

5. Chang, D. C. and G. J. Weng. 1979. "Elastic Moduli of Randomly Orientated, Chopped-Fiber Composites with Filled Resin", J. Material Sci., 14:2182.

6. Pipes, R. B., R. L. McCullough and D. G. Taggart. 1982. "Behavior of Discontinuous Fiber Composites: Fiber Orientation". Polymer Composites, 3(1):34-39.

7. Bozarth, J. M.. J. W. Gillespic and R. L. McCullough. 1987. "Fiber Orientation and Its Effect upon Thermoplastic Properties of Short Fiber Reinforced Poly(etheretherketone) (PEEK)", Polymer Composites, 8(2):74-81.

8. Pearson, J. R. A. 1983. Mechanics of Polymer Processing. Washington: McGraw-Hill (Hemisphere).

9. Middleman, S. 1977. Fundamentals of Polymer Processing. McGraw-Hill.

10. 1983. Computational Analvsis of Polymer Processing. J. R. A. Pearson and S. M. Richardson, eds. London. New York: Applied Science Publishers.

11. Isayev, A. 1. 1987. Injection and Compression Molding Fundamentals. New York: Marcel Dekker.

12. 1987. Applications of Computer Aided Engineering in Injection Molding. L. T. Manzione, ed. Hanser Publishers.

13. 1989. Fundamentals of Computer Modelling for Polymer Processing. C. L. Tucker, ed. Hanser Publishing Company.

14. Macosko, C. W. 1989. RIM Fundamentals of Reaction Injection Molding. Hanser Publishers.

15. Hele-Shaw. 1898. "The Flow of Water", Nature, 58:34-36.

16. Saffman, P. G. and G. Taylor. 1958. "The Penetration of a Fluid into a Porous Medium of HeleShaw Cell Containing a More Viscous Liquid", Proc. Roval Soc., A245:312-329.

17. Taylor, G. and P. G. Saffman. 1959. "A Note on the Motion of Bubbles in a Hele-Shaw Cell and Porous Medium", Quart. J. Mech. Appl. Math., 12:265-279.

18. Spencer, R. S. and G. D. Gilmore. 1950. "Residual Strains in Injection Molded Polystyrene". Mod. Plastics, 28:97-155.

19. Spencer, R. S. and G. D. Gilmore. 1951. "Some Flow Phenomena in the Injection Molding of Polystyrene", J. Colloid Sci., 6:118.

20. Gilmore, G. D. and R. S. Spencer. 1951. "Photographic Study of the Polymer Cycle in Injection Molding", Mod. Plastics, 28:117-185. 
21. Richardson, S. 1972. "Hele-Shaw Flows with a Free Boundary Produced by the Injection of a Fluid into a Narrow Channel", J. Fluid Mech., 56:609-618.

22. Beyer. C. E. and R. S. Spencer. 1960. Rheology, Theory and Appl, Vol. 3. New York: Academic Press.

23. Wu, P. C., C. F. Huang and C. G. Gogos. 1974. "Simulation of Mold Filling Process", Polymer Eng. Sci, 14:223-230.

24. Broyer, E., C. Gutfinger and $Z$. Tadmor. 1975. "A Theoretical Model for the Cavity Filling Process in Injection Molding", Trans. Sox. Rheology, 19:423-444.

25. Kamal, M. R., Y. Kuo and P. H. Doan. 1975. "The Injection Molding Behavior of Thermoplastics in Thin Rectangular Cavities", Polymer Eng. Sci., 15:863.

26. Kou. Y. and M. R. Kamal. 1976. "The Fluid Mechanics and Heat Transfer of Injection Mold Filling of Thermoplastic Materials", AlChE Journal, 22:661-669.

27. Oda, K., J. L. White and E. S. Clark. 1976. "Jetting Phenomena in Injection Molding", Polymer Eng. Sci., 16:585-592.

28. Bakerdjian, Z. and M. R. Kamal. 1977. "Distribution of Some Physical Properties in InjectionMolded Thermoplastic Parts", Polymer Eng. Sci., 17:96-100.

29. Gogos, C. G., C. F. Huang and L. Schmidt. 1986. "The Process of Cavity Filling Including the Fountain Flow in Injection Molding", Polymer Eng. Sci., 26(20):1457-1466.

30. Hieber, C. A. and S. F. Shen. 1978. "Flow Analysis of the Non-Isothermal Two-Dimensional Filling Process in Injection Molding", Israel J. Tech., 16:248-254.

31. Hieber, C. A. and S. F. Shen. 1980. "A Finite Element/Finite Difference Simulation of the Injection Mold Filling Process", J. Non-Newtonian Fluid Mech., 7:1-32.

32. Trafford, D. L., S. I. Güçeri and M. J. Crochet. "A Numerical Grid Generation Approach to the Injection Molding Problem", in NUMIFORM-Int. Conf. Numerical Methods in Industrial Forming, Gotenburg, Sweden, 1988.

33. Subbiah, S. D. L. Trafford and S. I. Güçeri. 1989. "Non-Isothermal Flow of Polymers into Two-Dimensional, Thin Cavity Molds: A Numerical Grid Generation Approach”, Int. J. Heat and Mass Transfer, 32:415-434.

34. Subbiah. S. 1989. "A Numerical Analysis of the Filling of Irregular Thin Cavities with NonIsothermal, Non-Newtonian Fluids", Ph.D. thesis, University of Delaware.

35. Couniot, A. and M. J. Crochet. "Finite Elements for the Numerical Simulation of Injection Molding", Proc. of the 2nd Intnl. Conf. on Numerical Methods for Forming Processes, 1986.

36. Behrens, R. A., M. J. Crochet, C. D. Denson and A. B. Metzner. 1987. "Transient Free-Surface Flows: Motion of a Fluid Advancing in a Tube". A/ChE Journal, 33(7):1168.

37. Coyle, D. J., J. W. Blake and C. W. Macosko. 1987. "The Kinematics of Fountain Flow in MoldFilling", AIChE Journal, 33(7):1178.

38. Blake, J. W. 1987. "Studies in Reaction Injection Mold Filling", Ph.D. Thesis, University of Minnesola.

39. Beris, A. N. 1987. "Fluid Elements Deformation behind an Advancing Flow Front", J. Rheology, 31:121-124.

40. Mavridis, H., A. N. Hrymak and J. Vlachopoulos. 1988. "The Effect of Fountain Flow on Molecular Orientation in Injection Molding", J. Rheology, 32(6):639-663.

41. Woebcken, W. 1962. "Effects of Processing Techniques on Structure of Molded Parts", Modern Plastics, (December): 146-161.

42. Belf, J. P. 1969. "Flow Orientation of Short Fiber Composites", J. Compos. Mat., 3:244-253.

43. Tadmor, Z. 1974. "Molecular Orientation in Injection Molding", J. Appl. Polymer Sci., 18: 1753-1772.

44. Darlington, M. W., P. L. McGinley and G. R. Smith. 1976. "Structure and Anisotropy of Stiffness in Glass Fibre-Reinforced Thermoplastics", J. Material Sci. , 11:877-886.

45. Gandhi, K. S. and R. Burns. 1976. "Rheological Properties of Glass Fiber-Reinforced Dough Molding Compounds", Trans. Soc: Rheology', 20(4):489-502. 
46. Owen, M. J. and K. Whybrew. 1976. "Fiber Orientation and Mechanical Properties in Polyester Dough Moulding Compounds (DMC)", Plastics and Rubber, l(6):231-238.

47. Chan, Y., J. L. White and Y. Oyanagi. 1976. "Influence of Glass Fibers on the Extrusion and Injection Molding Characteristics of Polyethylene and Polystyrene Melss", Polymer Eng. Sci., 18(4):268-272.

48. Bright, P. F., R. J. Crowson and M. J. Folkes. 1978. "A Study of the Effect of Injection Speed on Fiber Orientation in Simple Mouldings of Short Glass Fibre-Filled Polypropylene", Journal of Materials Science, 13:2497-2506.

49. Goettler, L. A. 1984. "Mechanical Property Enhancement in Short-Fiber Composites through the Control of Fiber Orientation during Fabrication". Polymer Composites, 5(1):60-71.

50. Sanou, M., B. Chung and C. Cohen. 1985. "Glass Fiber-Filled Thermoplastics. II. Cavity Filling and Fiber Orientation in Injection Molding". Polymer Eng. and Sci., 25(16):1008-1016.

51. Kenig, S. 1986. "Fiber Orientation Development in Molding of Polymer Composites", Polymer Composites, 7(1):50-55.

52. Vincent, M. and J. F. Agassant. 1986. "Experimental Study and Calculations of Short Glass Fiber Orientation in a Center Gated Molded Disc", Polymer Composites, 7(2):76-83.

53. Blanc, R., S. Philipon, M. Vincent, J. F. Agassant, H. Alglave, R. Müller and D. Froelich. 1987. "Injection Molding of Reinforced Thermosets: Fiber Orientation Observations and Flow Calculation", International Polymer Processing, 2:21-27.

54. Malik, T. M. and R. E. Prud'homme. 1986. "Determination of the Orientation of Mica in Composites", Polymer Composites, 7(5):315-322.

55. Monge, G., M. Vincent and J. Haudin. 1987. "Determination of Mineral Filler Orientation in Reinforced Thermoplastics by X-Ray Diffraction", Makromol. Chem., Macromol. Symp., 7(5): 315-322.

56. Advani, S. G. and C. L. Tucker. 1987. "The Use of Tensors to Describe and Predict Fiber Orientation in Short Fiber Composites", J. Rheology, 31(8):751-784.

57. Jeffery, G. B. 1922. "The Motion of Ellipsoidal Particles Immersed in a Viscous Fluid", Proc: Royal Soc, Al02:161-179.

58. Giesekus, H. 1962. "Elasto-Viskose Flüssigkeeiten für die in Stationären Schichtströmungen Sämtliche Normalspannungskomponenten Verschieden Grob Sind., Rheol. Acta, 2:50.

59. Bretherton, F. P. 1962. "The Motion of Rigid Particles in a Shear Flow at Low Reynolds Number", J. Fluid Mech., 14:284-304.

60. Brenner, H. 1964. "The Stokes Resistance of an Arbitrary Particle-II. An Extension", Chem. Eng. Sci., 19:599-629.

61. Chaffey, C. E., M. Takano and S. G. Mason. 1965. "Particle Motions in Sheared Suspensions, XVI. Orientations of Rods and Disks in Hyperbolic and Other Flows", Can. J. Phys., 43: 1269-1287.

62. Chaffer, C. E. and S. G. Mason. 1964. "Particle Bchavior in Shear and Electric Fields, III. Rigid Spheroids and Discs", J. Colloid Sci., 19:525-548.

63. Chaffer, C. E. and S. G. Mason. 1965. "Particle Behavior in Shear and Electric Fields, IV. The Viscosity of Suspensions of Non-Rotating Ellipsoids". J. Colloid Sci., 20:330-340.

64. Okagawa, A., R. G. Cox and S. G. Mason. 1973. "The Kinetics of Flowing Dispersions, VI. Transient Orientation and Rheological Phenomena of Rods and Discs in Shear Flow", J. Colloid Interface Sci., 45:303-329.

65. Okagawa, A. and S. G. Mason. 1973. "The Kinetics of Flowing Dispersions, VII. Oscillatory Behavior of Rods and Discs in Shear Flow", J. Colloid Interface Sci., 45:330-358.

66. Goldsmith. H. and S. G. Mason. 1967. Rheology: Theory and Applications. Academic Press. p. 85 .

67. Vincent, M. and J. F. Agassant. 1985. "Experimental and Theoretical Study of Short Fiber Orientation in Diverging Flows", Rheologica Acta, 24:603-610. 
68. Givler, R. C.. M. J. Crochet and R. B. Pipes. 1983. "Numerical Prediction of Fiber Orientation in Dilute Suspensions", J. Composite Materials. 17:330-343.

69. Givler, R. C. 1983. "Numerical Techniques for the Prediction of Flow Induced Orientation", Technical Report CCM-83-11. Center for Composite Materials, University of Delaware. Newark, DE 19716.

70. Givler, R. C. 1985. “Numerically Predicted Fiber Orientations in Dilute Suspensions", Ph.D. thesis, University of Delaware.

71. Hirsch, A. E. 1987. "Applied Fiber Orientation Prediction in Injection Molding", in 42nd Annual Technical Conference, Society of Plastics Engineers, Los Angeles, California, pp. 297299.

72. Squire, K. R., C. L. Vorres, D. E. Yuhas and C. S. Lee. 1987. "Experimental Verification of Fiber Orientation Predicted by Computer Simulation", in 47nd Annual Technical Conference, Society of Plastics Engineers, New York, New York, pp. 253-256.

73. Balasubramanyam. R. 1989. "Numerical Prediction of the Shape of a Fibre in Flowing Concentrated Suspensions", Composites, 20(1):14-19.

74. Goddard, J. D. and Y. H. Huang. 1983. "On the Motion of Flexible Threads in a Stoke's Shear Field", J. Non-Newtonian Fluid Mech., 13:47-62.

75. Folgar, F. and C. L. Tucker. 1984. "Orientation Behavior of Fibers in Concentrated Suspensions", J. Reinforced Plastics and Composites, 3:98-119.

76. Portillo, F. Folgar. 1983. "Fiber Orientation Distribution in Concentrated Suspensions: A Predictive Model", Ph.D. thesis. University of Illinois at Urbana-Champaign.

77. Jackson, W. C., S. G. Advani and C. L. Tucker. 1986. "Predicting the Orientation of Short Fibers in Thin Compression Moldings", J. Composite Materials, 20:539-557.

78. Matsuoka, T., J. Takabatake, Y. Inoue and H. Takahashi. 1990. "Prediction of Fiber Orientation in Injection Molded Parts of Short Fiber-Reinforced Thermoplastics", Polymer Eng Sci, 30(16): 957-966.

79. Doi, M. and S. F. Edwards. 1988. The Theory of Polymer Dynamics. Oxford Sci. Pub.

80. Doi. M. and S. F. Edwards. 1978. "Dynamics of Rodlike Macromolecules in Concentrated Solution. Part I", Journal of Chemical Society Faraday Transactions 1I, 74:560-570.

81. Doi, M. and S. F. Edwards. 1978. "Dynamics of Rodlike Macromolecules in Concentrated Solution, Part 2", Journal of Chemical Society Faraday Transactions II, 74:918-932.

82. Doi, M. 1981. "Molecular Dynamics and Rheological Properties of Concentrated Solutions of Rodlike Polymers in Isotropic and Liquid Crystalline Phases", J. Polym. Sci., Polym. Phys. Ed.

83. Doraiswamy, D. 1987. "Rheology of Polymeric Liquid Crystals", Ph.D. thesis, University of Delaware.

84. Doraiswamy, D. and A. B. Metzner. 1986. "The Rheology of Polymeric Liquid Crystals", Rheol. Acta, 25:580.

85. Metzner, A. B. and G. M. Prilutski. 1986. "Rheological Properties of Polymeric Liquid Crystals", J. Rheology, 30:663-691.

86. Marucci, G. 1981. "Prediction of Leslie Coefficients for Rodlike Polymer Nematics", Mol. Cryst. Liq. Cryst., 72:153-161.

87. Edwards, B. J. and A. N. Beris. 1989. "Flow-Induced Orientation in Monodomain Systems of Polymeric Liquid Crystals", J. Rheology, 33:537.

88. Becraft, M. 1988. "The Rheology of Concentrated Fiber Suspensions", Ph.D. thesis, University of Delaware.

89. Anzelius, A. 1931. "Über die bewegung der anisotropen flüssigkeiten", The Annual of the University of Upsala.

90. Oseen, C. W. 1933. "The Theory of Liquid Crystals", Faraday Soc. Trans., 29:883-899.

91. Frank, F. C. 1958. "1. Liquid Crystals: On the Theory of Liquid Crystals", Faraday Diss. Chem. Soc, , 25:19-28.

92. Ericksen, J. L. 1960. "Transversely Isotropic Fluids", Kolloid-Z., 173:117-122. 
93. Ericksen, J. L. 1960. “Anisotropic Fluids”, Arch. Rational Mech. Anal., 3-4:231-237.

94. Ericksen, J. L. 1961. "Poiseuille Flow of Certain Anisotropic Fluids", Arch. Rational Mech. Anal. , 8:1-8.

95. Ericksen, J. L. 1966. "Inequalities in Liquid Crystal Theory", Physics of Fluids, 9:1205-1207.

96. Ericksen, J. L. 1975. "General Solutions of the Hydrostatic Theory of Liquid Crystals", Trans. Soc. Rheology, 11:5-14.

97. Ericksen, J. L. 1976. "Theory of Liquid Crystals", in Adiances in Liquid Crystals, Vol. 2. pp. 233-298.

98. Leslie, F. M. 1966. "Some Constitutive Equations for Liquid Crystals", Q. J. Mech. Appl. Math., 19:357-370.

99. Leslie, F. M. 1968. "Some Constitutive Equations for Liquid Crystals", Arch. Rational Mech. Anal., 28:265-283

100. Leslie, F. M. 1979. "Theory of Flow Phenomena in Liquid Crystals", in Advances in Liquid Crystals, Vol. 4. pp. 1-81.

101. Leslie, F. M. 1981. "Viscosity of Nematic Liquid Crystals", Molecular Crystals and Liquid Crystals, 63:111-127.

102. Hand, G. L. 1962. "A Theory of Anisotropic Fluids", J. Fluid Mech., 13:33.

103. Duffy, R. B. 1978. "Flow of a Liquid with an Anisotropic Viscosity Tensor", J. Non-Newtonian Fluid Mech. , 4:177.

104. Viola, G. G. and D. G. Baird. 1986. "Studies on the Transient Shear Flow Behavior of Liquid Crystalline Polymers", J. Rheology. 30:601-628.

105. Duffy. R. B. 1988. "Orientation Effects in Extensional and Squeczing Flows of Certain Anisotropic Fluids", J. Non-Newtonian Fluid Mech., 28:77-97.

106. Batchelor, G. K. 1970. “The Stress System in a Suspension of Force-Free Particles", J. Fluid Mech., 41(3):545-570.

107. Batchelor, G. K. 1971. "The Stress Generated in a Non-Dilute Suspension of Elongated Particles by Pure Straining Motion," Journal of Fluid Mechanics, 46(4):813-829.

108. Dinh, S. M. and R. C. Armstrong. 1984. "A Rheological Equation State for Semiconcentrated Fiber Suspensions", J. Rheology, 28(3):207-227.

109. Dinh, S. M. 1981. "On the Rheology of Concentrated Fiber Suspensions". Sc. D. thesis, Massachusetts Institute of Technology.

110. Shaqfeh, E. S. and G. H. Fredrickson. 1990. "The Hydrodynamic Stress in a Suspension of Rods". Phys. Fluids A, 2(1):7-24.

111. Bibbo, M. A., S. M. Dinh and R. C. Armstrong. 1985. "Shear Flow Properties of SemiConcentrated Fiber Suspensions", J. Rheology, 29:905-929.

112. Ganani, E. and R. L. Powell. 1986. "Rheological Properties of Rodlike Particles in a Newtonian and a Non-Newtonian Fluid", J. Rheology. 30:995-1013.

113. Shanker, R., J. W. Gillespie and S. I. Guceri. 1990. "Rheology of Fiber Suspensions in Nonhomogeneous Flow Fields: Translation Induced Stresses". in Computer Modeling and Simulation of Manufacturing Processes. B. Singh. Y. T. Im. I. Haque and M. C. Altan. eds. ASME. $M D-V o l .20$ and $P E D-V o l .48$, pp. 267-272.

114. Papanastasiou. T. C. and A. N. Alexandrou. 1987. "Isothermal Extrusion of Non-Dilute Fiber Suspensions". J. Non-Newtonian Fluid Mech. , 25:313-328.

115. Lispcomb, G. G., M. M. Denn, D. U. Hur and D. V. Boger. 1988. "The Flow of Fiber Suspensions in Complex Geometries", J. of Non-Newronian Fluid Mech. , 26:297-325.

116. Evans, J. G. 1975. "The Flow of a Suspension of Force-Free Rigid Rods in a Newtonian Fluid". Ph.D. thesis, Cambridge University.

117. Hinch, E. J. and L. G. Leal. 1972. "The Effect of Brownian Motion on the Rheologica! Properties of a Suspension of Non-Spherical Particles", J. Fluid Mech., 52(4):683-712. 
118. Hinch. E. J. and L. G. Lcal. 1973. "Time-Dependent Shear Flows of a Suspension of Particles with Weak Brownian Rotations", J. Fluid Mech. . 57(4):753-767.

119. Chiba, K., K. Nakamura and D. V. Boger. 1990. "A Numerical Solution for the Flow of Dilute Fiber Suspensions through an Axisymmetric Contraction". J. Non-Newtonian Flaid Mech., $35: 1-14$.

120. Rosenberg, J., M. Denn and R. Keunings. 1990. "Simulations of Non-Recirculating Flows of Dilute Fiber Suspensions," J. Non-Newtonian Fluid Mech. . 37:317-345.

121. Altan, M. C., S. I. Güçeri and R. B. Pipes. "Anistropic Channel Flow of Fiber Suspensions", J. Non-Newtonian Fluid'Mech. (submitted for publication).

122. Gillespie, J. W. and J. A. Vanderschuren. 1985. "Process-Induced Fiber Orientation: Numerical Simulation with Experimental Verification”, Polimer Composites, 6:82-86.

123. Tucker, C. L. "Flow Regimes for Fiber Suspensions in Narrow Gaps". J. Non-Newtonian Fluid Mech. (submitted for publication).

124. Altan, M. C., S. Subbiah. S. I. Güçeri and R. B. Pipes. 1990. "Numerical Prediction of ThreeDimensional Fiber Orientation in Hele-Shaw Flows". Polym. Eng. Sci., 30:848-859.

125. Altan, M. C., S. G. Advani, S. I. Güçeri and R. B. Pipes. 1989. "On the Description of the Orientation State for Fiber Suspensions in Homogeneous Flows", J. Rheology, 33(7):1129-1155.

126. Altan, M. C. 1989. "Rheology of Fiber Suspensions and Fiber Orientation Analysis in Flow Processes", Ph.D. thesis, University of Delaware.

127. Friedrichs, B. S. I. Güçeri, S. Subbiah and M. C. Altan. 1990. "Simulation and Analysis of Mold Filling Processes with Polymer-Fiber Composites," in Processing of Polvmers and Polymeric Composites. A. A. Tseng and S. K. Soh, eds. ASME, MD-Vol. 19, pp. 73-90. 